\title{
RATIONAL CURVES ON HYPERSURFACES
}

\author{
B. WANG \\ AUG 20, 2014
}

\begin{abstract}
In this paper, we prove three related results;
(1) Extension of our result in [10] to all generic hypersurfaces. More precisely, the normal sheaf of a generic rational map $c_{0}$ to a generic hypersurface $X_{0}$ of $\mathbf{P}^{n}, n \geq 4$ has a vanishing higher cohomology,

$$
H^{1}\left(N_{c_{0} / X_{0}}\right)=0
$$

As applications we give

(2) A solution to a Voisin's conjecture [9] on a covering of a generic hypersurface by rational curves

(3) A classification of rational curves on hypersurfaces of general type-a solution to another Voisin's conjecture [9].
\end{abstract}

\section{Introduction}

\subsection{Statement}

We work over complex numbers, $\mathbb{C}$. A general or generic hypersurface is referred to a hypersurface as a point in a complement of a countable union of proper closed subsets of the space of all hypersurfaces. Let $\mathcal{L}=\mathcal{O}_{\mathbf{P}^{n}}(1)$, and

$$
X_{0}=\operatorname{div}\left(f_{0}\right)
$$

where $f_{0} \in H^{0}\left(\mathcal{L}^{h}\right)$ is generic. Let

$$
c_{0}: \mathbf{P}^{1} \rightarrow C_{0} \subset X_{0} \subset \mathbf{P}^{n}
$$

be birational onto an irreducible rational curve $C_{0}$. Let $N_{c_{0} / X_{0}}$ be the normal sheaf of the birational morphism $c_{0} 11$ Recall that it is a sheaf uniquely determined by the exact sequence of sheaves over $\mathbf{P}^{1}$,

$$
0 \rightarrow T_{\mathbf{P}^{1}} \stackrel{\left(c_{0}\right)^{*}}{\rightarrow} c_{0}^{*}\left(T_{X_{0}}\right) \rightarrow N_{c_{0} / X_{0}} \rightarrow 0
$$

where $\left(c_{0}\right)_{*}$, the differential of $c_{0}$ is an injective morphism of sheaf modules.

Theorem 1.1. (Main Theorem). Assume $n \geq 4$. Also assume that $X_{0}$ is a generic hypersurface containing $C_{0}$, and $c_{0}$ is generic in an irreducible component of $\operatorname{Hom}\left(\mathbf{P}^{1}, X_{0}\right)$. Then

$$
H^{1}\left(N_{c_{0} / X_{0}}\right)=0
$$

\footnotetext{
${ }^{1}$ Throughout the paper, one should be cautious about a fact that $c_{0}^{*}\left(T_{X_{0}}\right)$ is a locally free sheaf, but $N_{c_{0} / X_{0}}$ may not be. Thus the notation $N_{c_{0} / X_{0}}$ is always refereed to a sheaf module, the normal sheaf of the morphism.
} 


\subsection{Applications}

The main application of the result is in the area of rational curves on hypersurfaces. General hypersurfaces $X_{0}$ of degree $h$ in $\mathbf{P}^{n}$ can be divided into three different classes:

(1) Fano variety, in that case $h<n+1$ and there are lots of rational curves of each degree,

(2) Calabi-Yau variety, in that case $h=n+1$ and there are "fewer" rational curves of each degree,

(3) a variety of general type, in that case $h>n+1$ and there are no rational curves for large $h$.

A general question: what are the structures of the Hilbert schemes $\operatorname{Hilb}\left(X_{0}\right)$ of rational curves on $X_{0}$ ?

For the class (1), there are results of J. Harris, M. Roth and J. Starr (HRS) in [4](using different techniques from us), and a conjecture about Hilbert scheme Hilb $_{d}\left(X_{0}\right)$ of rational curves of degree $d$ by Coskun, Harris and Starr, who extend the results in [4] to almost all Fano hypersurfaces. For the classes (2), (3), the situations are quite different. However in a view of deformation theory, $H^{1}\left(N_{c_{0} / X_{0}}\right)$ is the obstruction space of the deformation of $c_{0}$ on $X_{0}$ if the rational map $c_{0}$ is smooth. So we try to identify the cohomology $H^{1}\left(N_{c_{0} / X_{0}}\right)$ as a unified invariant. In this view the result,

$$
H^{1}\left(N_{c_{0} / X_{0}}\right)=0 \text {. }
$$

has many implications. For examples if $c_{0}$ is a smooth embedding, it implies that

(1) deformation of $c_{0}$ on $X_{0}$ has no obstruction,

(2) deformation of $c_{0}$ over the complex moduli of $X_{0}$ has no obstruction.

These are immediate consequences of theorem 1.1. They all assert Hilbert schemes above have expected dimensions. More directly propositions 1.2, 1.3 below (or theorem 1,1), in the case $n \geq 4$, can be used to reproduce almost all results in [4], and furthermore to prove the conjecture made by Coskun, Harris and Starr 2. This will address the question for class (1). However the detailed discussion of this will be given elsewhere. In this paper we'll only concentrate on the classes (2), (3). Most of the known work and conjectures in this part (Classes (2), (3)) were nicely summarized by Voisin in [9]. In [9], Voisin studied the rational curves and elliptic curves on a variety $X_{0}$. As consequences she formulated many interesting conjectures. Here we list two of them.

(I) In a general Calabi-Yau hypersurface of dimension $\geq 3$, rational curves cover a countable union of Zariski closed proper algebraic subsets of codimension $\geq 2$.

(II) If a generic hypersurface is of general type, the degrees of rational curves on it are bounded.

In this paper, we apply theorem 1.1 to show that Voisin's conjectures (I), (II) are correct.

\subsection{Outline of the proof of Main theorem}

There is an easy reduction (see section 2.2) that shows that it suffices to prove theorem 1.1 for the Calabi-Yau hypersurface $X_{0}$, i.e.

$$
n+1=h .
$$

\footnotetext{
2It can't be directly used to prove the irreducibility of $\operatorname{Hilb}_{d}(X)$ for $X$ in class (1) (as proved and conjectured). Besides, the irreducibility in [4] clearly can not extend to classes (2), (3), while all the other results can.
} 
Let's outline the proof for this Calabi-Yau's case. Let $S=\mathbf{P}\left(H^{0}\left(\mathcal{O}_{\mathbf{P}^{n}}(h)\right)\right)$ be the space of all hypersurfaces of degree $h$. Theorem 1.1 is stated in terms of rational curves and hypersurfaces in projective space. But in this paper we'll stick with the affine space for the simplicity. So let

$$
\mathbb{C}^{(n+1)(d+1)}
$$

be the vector space,

$$
\left(H^{0}\left(\mathcal{O}_{\mathbf{P}^{1}}(d)\right)^{\oplus n+1}\right.
$$

whose open subset parametrizes the set of maps

$$
\mathbf{P}^{1} \rightarrow \mathbf{P}^{n}
$$

whose push-forward cycles have degree $d 3^{3}$ Throughout the paper, we let

$$
M=\mathbb{C}^{(n+1)(d+1)} .
$$

Let $M_{d}$ be the subset that consists of all birational-to-its-image maps $c$ whose pushforward cycles $c_{*}\left(\left[\mathbf{P}^{1}\right]\right)$ have degree $d . M$ has affine coordinates. Assume $c_{0}^{*}\left(f_{0}\right)=0$ for $c_{0} \in M_{d}$ as in theorem 1.1. Let $\mathbb{L} \subset S$ be an open set of the plane spanned by hypersurfaces $f_{0}, f_{1}, f_{2}$, where $f_{0}, f_{1}, f_{2}$ are generic in $S$. Let

$$
\Gamma_{\mathbb{L}} \ni\left(c_{0},\left[f_{0}\right]\right)
$$

be an irreducible component of the incidence scheme

$$
\left\{(c,[f]) \subset M \times \mathbb{L}: c^{*}(f)=0\right\}
$$

that is onto $\mathbb{L}$. We assume $\Gamma_{\mathbb{L}}$ exists. Let $P$ be the projection

$$
\Gamma_{\mathbb{L}} \rightarrow M
$$

The idea of the proof is to show that the scheme,

$$
P\left(\Gamma_{\mathbb{L}}\right) \subset M
$$

is a reduced, irreducible quasi-affine scheme of dimension $h+1$. The method is straightforward to show its defining polynomials at a generic point have non-degenerate Jacobian matrix (by that we mean it has full rank). See definition 2.6 below for the precise definition of a Jacobian matrix. All differentials and partial derivatives used throughout the paper are in algebraic sense (because all functions used are holomorphic). In the following we describe its defining polynomials and a differential form representing the Jacobian matrix.

Choose generic $h d+1$ distinct points $t_{i} \in \mathbf{P}^{1}$ (generic in $S y m^{h d+1}\left(\mathbf{P}^{1}\right)$ ). In the following (1.4), we use $t_{i}$ to denote a nonzero point in $\mathbb{C}^{2}$ with two coordinates $\left(\sigma_{1}^{i}, \sigma_{2}^{i}\right)$, i.e $P\left(\mathbb{C}^{2}\right)=\mathbf{P}^{1}$. Next we consider differential 1-forms $\phi_{i}$ on $M$ :

\footnotetext{
${ }^{3}$ The automorphism of $\mathbf{P}^{1}$ induces a $P G L(2)$ group action on $\mathbf{P}\left(\mathbb{C}^{(n+1)(d+1)}\right)$. Let $P G L(2)\left(c_{0}\right) \subset \mathbf{P}\left(\mathbb{C}^{h(d+1)}\right)$
}

be the orbit of $c_{0} \in \mathbf{P}\left(\mathbb{C}^{h(d+1)}\right)$. 


$$
\phi_{i}=d\left|\begin{array}{lll}
f_{2}\left(c\left(t_{i}\right)\right) & f_{1}\left(c\left(t_{i}\right)\right) & f_{0}\left(c\left(t_{i}\right)\right) \\
f_{2}\left(c\left(t_{1}\right)\right) & f_{1}\left(c\left(t_{1}\right)\right) & f_{0}\left(c\left(t_{1}\right)\right) \\
f_{2}\left(c\left(t_{2}\right)\right) & f_{1}\left(c\left(t_{2}\right)\right) & f_{0}\left(c\left(t_{2}\right)\right)
\end{array}\right|
$$

for $i=3, \cdots, h d+1$, and variable $c \in M$, where $|\cdot|$ denotes the determinant of a matrix. Notice $\phi_{i}$ are uniquely defined provided the quintics $f_{i}$ are in an affine open set of $S$ ( $\phi_{i}$ is not invariant under the $G L(2)$ action of $\mathbb{C}^{2}$ ). Let

$$
\omega=\wedge_{i=3}^{h d+1} \phi_{i} \in H^{0}\left(\Omega_{M}^{h d-1}\right)
$$

be the $h d-1$-form. This $\omega$ is the dual expression of the Jacobian matrix of some defining polynomials for the scheme $P\left(\Gamma_{\mathbb{L}}\right)$. The crucial point of this definition is that the polynomials inside of "d" operator,

$$
\left|\begin{array}{lll}
f_{2}\left(c\left(t_{i}\right)\right) & f_{1}\left(c\left(t_{i}\right)\right) & f_{0}\left(c\left(t_{i}\right)\right) \\
f_{2}\left(c\left(t_{1}\right)\right) & f_{1}\left(c\left(t_{1}\right)\right) & f_{0}\left(c\left(t_{1}\right)\right) \\
f_{2}\left(c\left(t_{2}\right)\right) & f_{1}\left(c\left(t_{2}\right)\right) & f_{0}\left(c\left(t_{2}\right)\right)
\end{array}\right|
$$

$i=3, \cdots, h d+1$ generically define the scheme (the scheme-theorectical under $P$ ),

$$
P\left(\Gamma_{\mathbb{L}}\right)
$$

Using successive blow-ups at a "boundary point" of $P\left(\Gamma_{\mathbb{L}}\right)$, we proved that

Proposition 1.2. For generic choices of quintics, $f_{0}, f_{1}, f_{2}$, and

$$
\left(t_{1}, \cdots, t_{h d+1}\right) \in \operatorname{Sym}^{h d+1}\left(\mathbf{P}^{1}\right),
$$

$\omega$ is a non-zero differential form of degree $h d-1$ when restricted to a non empty open set of $P\left(\Gamma_{\mathbb{L}}\right)$, i.e. the set of global sections $\left\{\phi_{i}\right\}_{i=3, \cdots, h d+1}$ is linearly independent in the $\mathcal{O}\left(P\left(\Gamma_{\mathbb{L}}\right)\right)$ module,

$$
H^{0}\left(\Omega_{M} \otimes \mathcal{O}_{P\left(\Gamma_{\mathbb{L}}\right)}\right)
$$

Next by an argument on Zariski tangent spaces, mainly from the fact that the ideal of scheme,

$$
P\left(\Gamma_{\mathbb{L}}\right)
$$

is generated by polynomials,

$$
\left|\begin{array}{lll}
f_{2}\left(c\left(t_{1}\right)\right) & f_{1}\left(c\left(t_{1}\right)\right) & f_{0}\left(c\left(t_{1}\right)\right) \\
f_{2}\left(c\left(t_{2}\right)\right) & f_{1}\left(c\left(t_{2}\right)\right) & f_{0}\left(c\left(t_{2}\right)\right) \\
f_{2}\left(c\left(t_{i}\right)\right) & f_{1}\left(c\left(t_{i}\right)\right) & f_{0}\left(c\left(t_{i}\right)\right)
\end{array}\right|
$$

we obtain

Proposition 1.3. If $\omega$ is non-zero on $P\left(\Gamma_{\mathbb{L}}\right)$ (The algebraic equivalence of this is that the set $\left\{\phi_{i}\right\}_{i=3, \cdots, h d+1}$ is linearly independent in the $\mathcal{O}\left(P\left(\Gamma_{\mathbb{L}}\right)\right)$ module $)$, then the Zariski tangent space of $P\left(\Gamma_{\mathbb{L}}\right)$ at a generic maximal point must be

$$
\operatorname{dim}(M)-\operatorname{deg}(\omega)
$$


Remark The form $\omega$ is not invariant under the $G L(2)$ action, but the zero locus $\{\omega=0\} \subset \mathbb{C}^{(n+1)(d+1)}$ is 4 The form $\omega$ depends on the generic choice of $t_{1}, \cdots, t_{h d+1}$, but $\{\omega=0\}$ does not. The proof of proposition 1.2 is the main body of the section 2. It is achieved by successive blow-ups at a rational curve lying on a product of $h$ distinct planes in general positions.

Continuing from this proposition, by the surjectivity of $\Gamma_{\mathbb{L}}$ to $\mathbb{L}$, the argument on Zariski tangent spaces shows that the dimension of Zariski tangent space of $\Gamma_{\mathbb{L}}$ at a generic point must be the same as that of Zariski tangent space of $P\left(\Gamma_{\mathbb{L}}\right)$ at a generic point. Thus the proposition 1.2 implies that the dimension of the Zariski tangent space of $\Gamma_{\mathbb{L}}$ at a generic point is $h+1$. By lemmas $2.8,2.9$, this directly leads to

$$
H^{1}\left(N_{c_{0} / X_{0}}\right)=0 .
$$

${ }^{4}$ The ideal of $\{\omega=0\}$ is a Jacobian ideal. 


\section{Proof of Main theorem}

The proof of theorem 1.1 in length is unbalanced with other sections. In order to be clear, we divided it into two steps that deal with two different types of hypersurfaces.

\subsection{Calai-Yau and Fano hypersurfaces}

The first case is $n+1-h \geq 0$. The hypersurfaces $X_{0}$ in this case is either Fano when $n+1-h>0$ or Calabi-Yau when $n+1-h=0$. Then we can repeat the proof in [10] to prove the main theorem. However some of the steps need to be altered from [10]. In order to insure the correctness of the proof, in the following we go through the proof of [10] step by step, and make changes when they are necessary. Also the proof for the Fano case is identical to that for the Calabi-Yau's. So in this subsection we only prove the case when $X_{0}$ is Calabi-Yau. Therefore we assume

$$
h=n+1 .
$$

We start with notations.

\subsubsection{Technical notations}

In this section, we collect all technical notations and definitions used in section 2.1. Some of them may already be defined before.

\section{Notations:}

(1) $S$ denotes the space all hypersurfaces of degree $h$, i.e. $S=\mathbf{P}\left(H^{0}\left(\mathcal{O}_{\mathbf{P}^{n}}(h)\right)\right)$.

Let $[f]$ denote the image of $f$ under the map

$$
H^{0}\left(\mathcal{O}_{\mathbf{P}^{n}}(h)\right)-\{0\} \quad \rightarrow \quad S .
$$

(2) Let

be

$$
\mathbb{C}^{(n+1)(d+1)} \simeq\left(H^{0}\left(\mathcal{O}_{\mathbf{P}^{1}}(d)\right)^{\oplus n+1}\right.
$$

and $M_{d}$ be the subset that parametrizes regular maps

$$
\mathbf{P}^{1} \rightarrow \mathbf{P}^{n}
$$

whose push-forward cycle has degree $d$.

(3) Throughout the paper, if

$$
c: \mathbf{P}^{1} \rightarrow \mathbf{P}^{n},
$$

is regular, $c^{*}(\sigma)$ denotes the pull-back section of section $\sigma$ of some bundle over $\mathbf{P}^{n}$. The vector bundles will not always be specified, but they are apparent in the context.

(4) Let $Y$ be a scheme, $y \in Y$ be a closed point, $Z \subset Y$ be a subscheme (open or closed) and $\mathcal{M}$ be a quasi-coherent sheaf of $\mathcal{O}_{Y}$-module. Then $\mathcal{O}_{y, Y}$ denotes the local ring, $\Omega_{Y}$ denotes the sheaf of differentials, $\left.\mathcal{M}\right|_{(Z)}$ denotes the pull-back sheaf module 
$i^{*}(\mathcal{M})$ where $i: Z \hookrightarrow Y$ is the embedding. We call $\left.\mathcal{M}\right|_{(Z)}$ the restriction of $\mathcal{M}$ to $Z$. $\left.\mathcal{M}\right|_{Z}$ denotes the localization of $\mathcal{M}$ at $Z$, which is a $\mathcal{O}_{Z, Y}$ module. Thus

$$
\left.\mathcal{M}\right|_{(\{y\})}=\left.\mathcal{M}\right|_{Z} \otimes k(y),
$$

where $k(y)$ is the residue field of the maximal point $\{y\}$.

If $Y$ is quasi-affine scheme, $\mathcal{O}(Y)$ denotes the ring of regular functions on $Y$.

(5) Let $\alpha \in T_{c_{0}} M$, and

$$
g: M \rightarrow H^{0}\left(\mathcal{O}_{\mathbf{P}^{1}}(r)\right)
$$

be a regular map. Then the image $g_{*}(\alpha)$ of $\alpha$ under the differential map at $c_{0}$ is denoted by

$$
\frac{\partial g\left(c_{0}(t)\right)}{\partial \alpha} \in T_{g\left(c_{0}\right)}\left(H^{0}\left(\mathcal{O}_{\mathbf{P}^{1}}(r)\right)\right)=H^{0}\left(\mathcal{O}_{\mathbf{P}^{1}}(r)\right) .
$$

(use the identification $\left.T_{g\left(c_{0}\right)}\left(H^{0}\left(\mathcal{O}_{\mathbf{P}^{1}}(r)\right)\right)=T_{0}\left(H^{0}\left(\mathcal{O}_{\mathbf{P}^{1}}(r)\right)\right)\right)$.

(6) If $Y$ is a scheme, $|Y|$ denotes the induced reduced scheme of $Y$.

DeFinition 2.1. Let

$$
c_{0}^{*}\left(T_{X_{0}}\right) \simeq \mathcal{O}_{\mathbf{P}^{1}}\left(a_{1}\right) \oplus \cdots \oplus O_{\mathbf{P}^{1}}\left(a_{n-1}\right) .
$$

where

$$
a_{1} \geq \cdots \geq a_{n-1}, \text { and } \sum_{i} a_{i}=0
$$

We'll fix an isomorphism in (2.2) throughout. Let $E$ be the pull-back of the summand $\sum_{a_{i} \geq 0} \mathcal{O}_{\mathbf{P}^{1}}\left(a_{i}\right)$ under the map

$$
c_{0}^{*}\left(T_{X_{0}}\right) \rightarrow N_{c_{0} / X_{0}}
$$

So $E$ is the sub-bundle of the bundle $c_{0}^{*}\left(T_{X_{0}}\right)$, generated by all the holomorphic sections of $c_{0}^{*}\left(T_{X_{0}}\right)$.

Definition 2.2. (a) If $f \in H^{0}\left(\mathcal{O}_{\mathbf{P}^{n}}(h)\right)$ is a degree $d$ polynomial other than $f_{0}$, we denote the direction of the line through two points $[f],\left[f_{0}\right]$ in the projective space, $\mathbf{P}\left(H^{0}\left(\mathcal{O}_{\mathbf{P}^{n}}(h)\right)\right.$ by $\vec{f}$. So

$$
\vec{f} \in T_{\left[f_{0}\right]} \mathbf{P}\left(H^{0}\left(\mathcal{O}_{\mathbf{P}^{n}}(h)\right) .\right.
$$

(b) Note that the vector $\vec{f}$ is well-defined up-to a non-zero multiple. In case when $c_{0}$ can deform to all hypersurfaces to the first order, i.e. the map in (2.7) below is surjective, this naturally gives a section $\left\langle\vec{f}>\right.$ of the bundle $c_{0}^{*}\left(T_{\mathbf{P}^{n}}\right)$ (may not be unique), to each deformation $\vec{f}$ of the hypersurface $f_{0}$. This is easily can be understood as the direction of the moving $c_{0}$ in the deformation $(\vec{f},<\vec{f}>)$ of the pair $\left(c_{0}, f_{0}\right)$.

DEFINITION 2.3. Let $\Gamma$ be an irreducible component of the incidence scheme

$$
\left\{(c, f) \subset M \times \mathbf{P}\left(H^{0}\left(\mathcal{O}_{\mathbf{P}^{n}}(h)\right)\right): c^{*}(f)=0\right\}
$$


that dominates $S=\mathbf{P}\left(H^{0}\left(\mathcal{O}_{\mathbf{P}^{n}}(h)\right)\right)$. Let $\left(c_{0},\left[f_{0}\right]\right) \in \Gamma$ be a generic point. Throughout the paper we assume that such a $\Gamma$ exists.

Remark: The existence of such a $\Gamma$ is equivalent to the assumption of theorem 1.1: $X_{0}$ is generic. Results in section 2.1.2 only need a weaker assumption, but the main propositions $1.2,1.3$ rely on this stronger assumption $-\Gamma$ exists. In this paper, to avoid the distraction, we use the unified and consistent assumption- $\Gamma$ exists.

Definition 2.4. Let $f_{1}, f_{2} \in H^{0}\left(\mathcal{O}_{\mathbf{P}^{n}}(h)\right)$ be two quintics different from $f_{0}$. Let $\mathbb{L}$ be an open set of the plane in

$$
\mathbf{P}\left(H^{0}\left(\mathcal{O}_{\mathbf{P}^{n}}(h)\right)\right)
$$

spanned by $\left[f_{0}\right],\left[f_{1}\right],\left[f_{2}\right]$ and centered around $\left[f_{0}\right]$.

DeFinition 2.5. Let

$$
\Gamma_{\mathbb{L}}=\Gamma \cap(M \times \mathbb{L})
$$

be an irreducible component of the restriction of $\Gamma$ to $M \times \mathbb{L}$ such that it is onto $\mathbb{L}$, and

$$
\Gamma_{f_{0}}, \text { for generic } f_{0} \in \mathbb{L}
$$

is an irreducible component of

$$
P\left(\Gamma \cap\left(M \times\left\{\left[f_{0}\right]\right\}\right)\right)
$$

where $P$ is the projection to $M$.

DEFINITION 2.6. Let $V$ be a smooth analytic variety with analytic coordinates $x_{1}, \cdots, x_{p}$, Let $f_{1}, \cdots, f_{m}$ be holomorphic functions on $V$. We define

$$
\left(\begin{array}{cccc}
\frac{\partial f_{1}}{\partial x_{1}} & \frac{\partial f_{1}}{\partial x_{2}} & \cdots & \frac{\partial f_{1}}{\partial x_{p}} \\
\frac{\partial f_{2}}{\partial x_{1}} & \frac{\partial f_{2}}{\partial x_{2}} & \cdots & \frac{\partial f_{2}}{\partial x_{p}} \\
\vdots & \vdots & \cdots & \vdots \\
\frac{\partial f_{m}}{\partial x_{1}} & \frac{\partial f_{m}}{\partial x_{2}} & \cdots & \frac{\partial f_{m}}{\partial x_{p}}
\end{array}\right) .
$$

to be the Jacobian matrix of functions $f_{1}, \cdots, f_{m}$. This Jacobian matrix depends on the coordinates $x_{1}, \cdots, x_{p}$.

We defined the matrix for the set of functions $f_{1}, \cdots, f_{m}$. One may wish to compare this definition with that of Jacobian ideals which is independent choice of those functions. 


\subsubsection{First order}

Let's start the problem in its first order.

Lemma 2.7. Let $f_{0}$ be a generic hypersurface containing a rational map $c_{0}$ as before. If $\left(c_{0},\left[f_{0}\right]\right) \in|\Gamma|$ is generic, then the projection

$$
T_{\left(c_{0},\left[f_{0}\right]\right)} \Gamma \stackrel{P^{s}}{\rightarrow} T_{\left[f_{0}\right]} S
$$

is surjective, where $S=\mathbf{P}\left(H^{0}\left(\mathcal{O}_{\mathbf{P}^{n}}(h)\right)\right.$.

Proof. Let $|\Gamma| \subset \Gamma$ be the reduced scheme of the scheme $\Gamma$. By the genericity of $f_{0}$, the projection

$$
|\Gamma| \rightarrow S
$$

is dominant. Hence in a neighborhood of a generic point $\left(c_{0},\left[f_{0}\right]\right) \in|\Gamma|$, the projection is a smooth map. Thus

$$
T_{\left(c_{0},\left[f_{0}\right]\right)}|\Gamma| \rightarrow T_{\left[f_{0}\right]} S
$$

is surjective. This proves the lemma

口

To elaborate definition 2.2, we apply this lemma to obtain that for any $\alpha \in T_{\left[f_{0}\right]} S$, there is a section denoted by

$$
<\alpha>\in H^{0}\left(c_{0}^{*}\left(T_{\mathbf{P}^{n}}\right)\right)
$$

such that

$$
(\alpha,<\alpha>)
$$

is tangent to the universal hypersurface

$$
\mathcal{X}=\{(x, f): x \in \operatorname{div}(f)\}
$$

in

$$
\mathbf{P}^{n} \times S
$$

Note that $\langle\alpha\rangle$ is unique up to a section in $H^{0}\left(c_{0}^{*}\left(T_{X_{0}}\right)\right)$. But we will always fix $\langle\alpha\rangle$ as in definition 2.2 .

\subsubsection{The incidence scheme}

In this subsection, we study the Zariski tangent spaces of various incidence schemes to reveal a connection between the incidence scheme and the normal sheaf.

Lemma 2.8. Let $\left[f_{0}\right] \in S$ be a generic point, $\mathbb{L}_{1} \subset S$ an open set of the pencil containing $f_{0}$ and another quintic $f_{1}$. Let $\left(c_{0},\left[f_{0}\right]\right) \in \Gamma_{\mathbb{L}_{1}}$ be generic. Then 
(a)

$$
\frac{T_{c_{0}} \Gamma_{f_{0}}}{k e r} \simeq H^{0}\left(c_{0}^{*}\left(T_{X_{0}}\right)\right)
$$

where ker is a line in $T_{c_{0}} \Gamma_{f_{0}}$.

$$
\operatorname{dim}\left(T_{\left(c_{0},\left[f_{0}\right]\right)} \Gamma_{\mathbb{L}_{1}}\right)=\operatorname{dim}\left(T_{c_{0}} \Gamma_{f_{0}}\right)+1
$$

and furthermore

$$
\operatorname{dim}\left(T_{c_{0}} P\left(\Gamma_{\mathbb{L}_{1}}\right)\right)=\operatorname{dim}\left(T_{c_{0}} \Gamma_{f_{0}}\right)+1
$$

Proof. (a). Let $a_{i}(c, f), i=0, \cdots, h d$ be the coefficients of polynomial $f(c(t))$ in parameter $t$. Then the scheme

\section{$\Gamma$}

in $M \times \mathbf{P}^{n}$ is defined by homogeneous polynomials

$$
a_{i}(c, f)=0, i=0, \cdots, h d, \text { locally. }
$$

Let $\alpha \in T_{c_{0}} M$. The equations

$$
\frac{\partial a_{i}\left(c_{0}, f_{0}\right)}{\partial \alpha}=0, \text { all } i
$$

by the definition, are necessary and sufficient conditions for $\alpha$ lying in

$$
T_{\left(c_{0},\left[f_{0}\right]\right)} \Gamma_{f_{0}} .
$$

On the other hand there is an evaluation map $e$ :

$$
\begin{aligned}
& M \times \mathbf{P}^{1} \rightarrow \mathbf{P}^{n} \\
& (c, t) \rightarrow c(t)
\end{aligned}
$$

The differential map $e_{*}$ gives a morphism $e_{m}$ :

$$
\begin{array}{ccc}
T_{c_{0}} M & \stackrel{e_{m}}{\rightarrow} & H^{0}\left(c_{0}^{*}\left(T_{\mathbf{P}^{n}}\right)\right) \\
\alpha & \rightarrow & e_{*}(\alpha)
\end{array}
$$

Suppose there is an $\alpha$ such that $e_{*}(\alpha)=0$. We may assume $c_{0}$ is a map

$$
\mathbb{C}^{1} \rightarrow \mathbb{C}^{n+1}-\{0\}
$$

Since $c_{0}$ is birational to its image, there is a Zariski open set

$$
U_{\mathbf{P}^{1}} \subset \mathbf{P}^{1}
$$

and an open set

$$
V \subset\left\{\left(c_{0}(t)\right)\right\} \subset \mathbb{C}^{n+1}-\{0\}
$$


such that $\left.c_{0}^{\prime}\right|_{U_{\mathbf{P} 1}}$ is an isomorphism

$$
U_{\mathbf{P}^{1}} \rightarrow V
$$

where $c_{0}^{\prime}$ is the map from $U_{\mathbf{P}^{1}}$ to $\mathbb{C}^{n+1}$ induced from $c_{0}$. Due to the equation $e_{*}(\alpha)=0$, on $T_{t} U_{\mathbf{P}^{1}}$

$$
\left(\alpha_{0}(t), \cdots, \alpha_{n}(t)\right)=\lambda(t) c_{0}(t)
$$

on $V$ (at each point $\left(c_{0}(t), \cdots, c_{n}(t)\right)$ of $V$ ) where $\lambda(t)$ lies in $\mathcal{O}\left(U_{\mathbf{P}^{1}}\right)$. Because $\left(\alpha_{0}(t), \cdots, \alpha_{n}(t)\right)$ is parallel to $c_{0}(t)$ at all points $t \in \mathbf{P}^{1}, \lambda$ can be extended to $\mathbf{P}^{1}$. Hence $\lambda(t)$ is in $\mathcal{O}\left(\mathbf{P}^{1}\right)$. So it is a constant (independent of $t$ ). Therefore $\alpha \in \mathbb{C}^{h(d+1)}$ is parallel to

$$
c_{0} \neq 0 \in \mathbb{C}^{h(d+1)} .
$$

This shows that

$$
\operatorname{dim}\left(\operatorname{ker}\left(e_{m}\right)\right)=1
$$

(this does not hold if $c_{0}$ is a multiple cover map). By the dimension count, $e_{m}$ must be surjective. For any $\alpha \in c_{0}^{*}\left(T_{\mathbf{P}^{n}}\right), \alpha \in c_{0}^{*}\left(T_{X_{0}}\right)$ if and only if

$$
\frac{\partial f_{0}\left(c_{0}(t)\right)}{\partial \alpha}=0,
$$

for generic $t \in \mathbf{P}^{1}$. Notice equations (2.13) and (2.16) are exactly the same. Therefore $e_{m}$ induces an isomorphism

$$
\frac{T_{c_{0}} \Gamma_{f_{0}}}{\operatorname{ker}\left(e_{m}\right)} \stackrel{e_{m}}{\rightarrow} H^{0}\left(c_{0}^{*}\left(T_{X_{0}}\right)\right)
$$

This proves part (a).

(b). Let $t_{1}, \cdots, t_{h d+1} \in \mathbf{P}^{1}$ be $h d+1$ distinct points of $\mathbf{P}^{1}$.

Notice $c^{*}(f)=0$ if and only if $\left.c^{*}(f)\right|_{t_{i}}=0$ for all $i$. Then

$$
\Gamma_{\mathbb{L}_{1}}
$$

is an irreducible component of

$$
\left\{(c, f) \in M \times \mathbb{L}_{1}:\left.c^{*}(f)\right|_{t_{i}}=0, i=1, \cdots, h d+1\right\} .
$$

surjective to $S$ in first order. Let $\alpha \in T_{c_{0}} M$ and $\beta=\vec{f}_{1}$. Let $\epsilon \in \mathbb{C}$, then $\epsilon \beta \in T_{\left[f_{0}\right]} \mathbb{L}_{1}$. Then the Zariski tangent space $T_{c_{0}} \Gamma_{\mathbb{L}_{1}}$ is

$$
\left\{(\alpha, \epsilon \beta) \in T_{\left(c_{0},\left[f_{0}\right]\right)}\left(M \times \mathbb{L}_{1}\right): \epsilon f_{1}\left(c_{0}\left(t_{i}\right)\right)+\frac{\partial f_{0}\left(c_{0}\left(t_{i}\right)\right)}{\partial \alpha}=0, i=1, \cdots, h d+1\right\}
$$

Because $\left[f_{0}\right]$ is a generic point of $\mathbb{L}_{1}$, the map (2.7) is surjective. Thus there is $\alpha_{0} \in T_{c_{0}}(M)$ such that

$$
f_{1}\left(c_{0}(t)\right)+\frac{\partial f_{0}\left(c_{0}(t)\right)}{\partial \alpha_{0}}=0
$$


for all $t \in \mathbf{P}^{1}$. Thus $T_{\left(c_{0},\left[f_{0}\right]\right)} \Gamma_{\mathbb{L}_{1}}$ is isomorphic to

$$
\left\{(\alpha, \epsilon) \in \mathbb{C}^{(n+1)(d+1)+1}: \frac{\partial f_{0}\left(c_{0}\left(t_{i}\right)\right)}{\partial\left(\alpha-\epsilon \alpha_{0}\right)}=0, i=1, \cdots, h d+1\right\} .
$$

Notice that the subspace with $\epsilon=0$,

$$
\left\{\alpha \in T_{c_{0}} M: \frac{\partial f_{0}\left(c_{0}\left(t_{i}\right)\right)}{\partial \alpha}=0, i=1, \cdots, h d+1\right\},
$$

is the tangent space of the scheme

$$
\Gamma_{f_{0}} .
$$

Thus the dimensions of them differ by 1 .

Next we prove the assertion for the scheme-theoretical image $P\left(\Gamma_{\mathbb{L}_{1}}\right)$. Using an expression of $\Gamma_{\mathbb{L}_{1}}, P\left(\Gamma_{\mathbb{L}_{1}}\right)$ is defined by polynomials

$$
f_{1}\left(c\left(t_{i}\right)\right) f_{0}\left(c\left(t_{j}\right)\right)-f_{1}\left(c\left(t_{j}\right)\right) f_{0}\left(c\left(t_{i}\right)\right)=0,1 \leq i, j \leq h d+1 .
$$

Assume non of $t_{i}, i=1, \cdots, h d+1$ is a zero of $f_{1}\left(c_{0}(t)\right)=0$. Then there exists an open set $U$ of $M$ around $c_{0}$ such that

$$
P\left(\Gamma_{\mathbb{L}_{1}}\right) \cap U
$$

is defined by $h d$ equations

$$
f_{1}\left(c\left(t_{h d+1}\right)\right) f_{0}\left(c\left(t_{j}\right)\right)-f_{1}\left(c\left(t_{j}\right)\right) f_{0}\left(c\left(t_{h d+1}\right)\right)=0, j=1, \cdots, h d .
$$

We'll denote $U$ by $M$. Then the Zariski tangent space $T_{c_{0}} P\left(\Gamma_{\mathbb{L}_{1}}\right)$ is defined by

$$
\begin{gathered}
f_{1}\left(c_{0}\left(t_{h d+1}\right)\right) \frac{\partial f_{0}\left(c_{0}\left(t_{j}\right)\right)}{\partial \alpha}-f_{1}\left(c_{0}\left(t_{j}\right)\right) \frac{\partial f_{0}\left(c_{0}\left(t_{h d+1}\right)\right)}{\partial \alpha}=0, \\
j=1, \cdots, h d .
\end{gathered}
$$

where $\alpha \in T_{c_{0}} M$. This is the same as

$$
\frac{\partial f_{0}\left(c_{0}\left(t_{j}\right)\right)}{\partial \alpha}-\frac{f_{1}\left(c_{0}\left(t_{j}\right)\right)}{f_{1}\left(c_{0}\left(t_{h d+1}\right)\right)} \frac{\partial f_{0}\left(c_{0}\left(t_{h d+1}\right)\right)}{\partial \alpha}=0
$$

Next we view $\frac{\partial f_{0}\left(c_{0}\left(t_{j}\right)\right)}{\partial \alpha}$ as an element in

$$
\left(T_{c_{0}} M\right)^{*} .
$$

If

$$
\frac{\partial f_{0}\left(c_{0}\left(t_{i}\right)\right)}{\partial \alpha}, i=1, \cdots, h d+1
$$

are linearly independent, then $\operatorname{dim}\left(T_{c_{0}} \Gamma_{f_{0}}\right)=(n+1-h) d+n$ and by $(2.23)$,

$$
\operatorname{dim}\left(T_{c_{0}} P\left(\Gamma_{\mathbb{L}_{1}}\right)\right)=(n+1-h) d+n+1 .
$$

The lemma is proved.

If

$$
\frac{\partial f_{0}\left(c_{0}\left(t_{i}\right)\right)}{\partial \alpha}, i=1, \cdots, h d+1
$$


are linearly dependent, there are two cases:

(1) All solutions $\alpha_{0}$ to $(2.23)$ satisfy

$$
\frac{\partial f_{0}\left(c_{0}\left(t_{h d+1}\right)\right)}{\partial \alpha}=0
$$

(2) Some solutions $\alpha_{0}$ to (2.23) do not satisfy

$$
\frac{\partial f_{0}\left(c_{0}\left(t_{h d+1}\right)\right)}{\partial \alpha}=0
$$

The case (1) is false. Because if

$$
\frac{\partial f_{0}\left(c_{0}\left(t_{h d+1}\right)\right)}{\partial \alpha_{0}}=0
$$

for all solution $\alpha_{0}$ of $(2.23)$, then $\frac{\partial f_{0}\left(c_{0}\left(t_{j}\right)\right)}{\partial \alpha_{0}}=0$ for all $j=1, \cdots, h d+1$. Hence all solutions $\alpha_{0}$ to (2.23) must be sections of $E$ (see definition 2.1 for $E$ ). As we know

$$
<\vec{f}_{1}>
$$

is a solution to $(2.23)$, but

$$
\frac{\partial f_{0}\left(c_{0}(t)\right)}{\partial<\vec{f}_{1}>}=-f_{1}\left(c_{0}(t)\right)
$$

which means $<\vec{f}_{1}>$ is not a section of $E$. This is a contradiction.

In case (2), the solutions $\alpha_{0}$ to (2.23) must either satisfy

$$
\frac{\partial f_{0}\left(c_{0}\left(t_{j}\right)\right)}{\partial \alpha}=0
$$

for all $j=1, \cdots, h d+1$, in which case, they are sections of $E$, or are uniquely expressed as

$$
\frac{\partial f_{0}\left(c_{0}\left(t_{j}\right)\right)}{\partial \alpha_{0}}=\frac{f_{1}\left(c_{0}\left(t_{j}\right)\right)}{f_{1}\left(c_{0}\left(t_{h d+1}\right)\right)} \frac{\partial f_{0}\left(c_{0}\left(t_{h d+1}\right)\right)}{\partial \alpha_{0}}, j=1, \cdots, h d+1
$$

which are exactly $<\vec{f}_{1}>$. Thus the vector $<\vec{f}_{1}>$ offers another dimension to

$$
\operatorname{dim}\left(T_{c_{0}} P\left(\Gamma_{\mathbb{L}_{1}}\right)\right) .
$$

So

$$
T_{c_{0}} P\left(\Gamma_{\mathbb{L}_{1}}\right)=T_{c_{0}} \Gamma_{f_{0}} \oplus \mathbb{C}<\vec{f}_{1}>_{M}
$$

where $<\vec{f}_{1}>_{M} \in e_{m}^{-1}\left(<\vec{f}_{1}>\right)$. The lemma is proved.

口

Now we can describe the case when this is based on 2 dimensional plane in $S$. Recall $\mathbb{L}$ is an open set of a plane spanned by $f_{0}, f_{1}, f_{2}$, and

$$
\mathbb{L}_{1} \subset \mathbb{L}
$$


is a pencil containing $\left[f_{0}\right]$.

Lemma 2.9. For generic $c_{g} \in P\left(\Gamma_{\mathbb{L}_{1}}\right) \subset P\left(\Gamma_{\mathbb{L}}\right)$,

$$
\operatorname{dim}\left(T_{c_{g}} P\left(\Gamma_{\mathbb{L}}\right)\right)=\operatorname{dim}\left(T_{c_{g}} P\left(\Gamma_{\mathbb{L}_{1}}\right)\right)+1
$$
$\mathbb{C}^{3}$

Proof. Consider an open set $U_{P\left(\Gamma_{\mathbb{L}}\right)}$ of $P\left(\Gamma_{\mathbb{L}}\right)$ centered at $c_{g}$ such that vectors in

$$
\left(\begin{array}{l}
\left.f_{2}\left(c\left(t_{1}\right)\right), f_{1}\left(c\left(t_{1}\right)\right), f_{0}\left(c\left(t_{1}\right)\right)\right) \\
\left.f_{2}\left(c\left(t_{2}\right)\right), f_{1}\left(c\left(t_{2}\right)\right), f_{0}\left(c\left(t_{2}\right)\right)\right)
\end{array}\right\}
$$

and vectors in $\mathbb{C}^{h d+1}$

$$
\left(\begin{array}{c}
f_{2}\left(c\left(t_{h d+1}\right)\right. \\
\vdots \\
f_{2}\left(c\left(t_{2}\right)\right) \\
f_{2}\left(c\left(t_{1}\right)\right)
\end{array}\right),\left(\begin{array}{c}
f_{1}\left(c\left(t_{h d+1}\right)\right. \\
\vdots \\
f_{1}\left(c\left(t_{2}\right)\right) \\
f_{1}\left(c\left(t_{1}\right)\right)
\end{array}\right)
$$

are linearly independent for all $c \in U_{P\left(\Gamma_{\mathbb{L}}\right)}$. If

$$
\begin{gathered}
\left|\begin{array}{lll}
f_{2}\left(c\left(t_{1}\right)\right) & f_{1}\left(c\left(t_{1}\right)\right) & f_{0}\left(c\left(t_{1}\right)\right) \\
f_{2}\left(c\left(t_{2}\right)\right) & f_{1}\left(c\left(t_{2}\right)\right) & f_{0}\left(c\left(t_{2}\right)\right) \\
f_{2}\left(c\left(t_{i}\right)\right) & f_{1}\left(c\left(t_{i}\right)\right) & f_{0}\left(c\left(t_{i}\right)\right)
\end{array}\right|=0 \\
\left(f_{2}\left(c\left(t_{i}\right)\right), f_{1}\left(c\left(t_{i}\right)\right), f_{0}\left(c\left(t_{i}\right)\right)\right)
\end{gathered}
$$

for all $i=1, \cdots, h d+1$ are linear combinations of

$$
\begin{aligned}
& \left(f_{2}\left(c\left(t_{1}\right)\right), f_{1}\left(c\left(t_{1}\right)\right), f_{0}\left(c\left(t_{1}\right)\right)\right) \\
& \left(f_{2}\left(c\left(t_{2}\right)\right), f_{1}\left(c\left(t_{2}\right)\right), f_{0}\left(c\left(t_{2}\right)\right)\right) .
\end{aligned}
$$

Hence

$$
\left|\begin{array}{lll}
f_{2}\left(c\left(t_{i}\right)\right) & f_{1}\left(c\left(t_{i}\right)\right) & f_{0}\left(c\left(t_{i}\right)\right) \\
f_{2}\left(c\left(t_{j}\right)\right) & f_{1}\left(c\left(t_{j}\right)\right) & f_{0}\left(c\left(t_{j}\right)\right) \\
f_{2}\left(c\left(t_{l}\right)\right) & f_{1}\left(c\left(t_{l}\right)\right) & f_{0}\left(c\left(t_{l}\right)\right)
\end{array}\right|=0
$$

for all $i, j, l$ between 1 and $h d+1$.

Since

$$
\left|\begin{array}{lll}
f_{2}\left(c\left(t_{i}\right)\right) & f_{1}\left(c\left(t_{i}\right)\right) & f_{0}\left(c\left(t_{i}\right)\right) \\
f_{2}\left(c\left(t_{j}\right)\right) & f_{1}\left(c\left(t_{j}\right)\right) & f_{0}\left(c\left(t_{j}\right)\right) \\
f_{2}\left(c\left(t_{l}\right)\right) & f_{1}\left(c\left(t_{l}\right)\right) & f_{0}\left(c\left(t_{l}\right)\right)
\end{array}\right|=0
$$


for all $i, j, l$ between 1 and $h d+1$ define $U_{P\left(\Gamma_{\mathbb{L}}\right)}, h d-1$ equations

$$
\left|\begin{array}{lll}
f_{2}\left(c\left(t_{1}\right)\right) & f_{1}\left(c\left(t_{1}\right)\right) & f_{0}\left(c\left(t_{1}\right)\right) \\
f_{2}\left(c\left(t_{2}\right)\right) & f_{1}\left(c\left(t_{2}\right)\right) & f_{0}\left(c\left(t_{2}\right)\right) \\
f_{2}\left(c\left(t_{i}\right)\right) & f_{1}\left(c\left(t_{i}\right)\right) & f_{0}\left(c\left(t_{i}\right)\right)
\end{array}\right|=0,
$$

for $i=3, \cdots, h d+1$ define the scheme $U_{P\left(\Gamma_{\mathrm{L}}\right)}$. Then by the definition there is $f_{g} \in \mathbb{L}$ such that

$$
c_{g}^{*}\left(f_{g}\right)=0 .
$$

We denote $U_{\mathbb{L}}$ by $\mathbb{L}$. Then $\left.P\left(\Gamma_{\mathbb{L}}\right)\right)$ is defined by the polynomial equations

$$
\left|\begin{array}{lll}
f_{2}\left(c\left(t_{i}\right)\right) & f_{1}\left(c\left(t_{i}\right)\right) & f_{0}\left(c\left(t_{i}\right)\right) \\
f_{2}\left(c\left(t_{1}\right)\right) & f_{1}\left(c\left(t_{1}\right)\right) & f_{0}\left(c\left(t_{1}\right)\right) \\
f_{2}\left(c\left(t_{2}\right)\right) & f_{1}\left(c\left(t_{2}\right)\right) & f_{0}\left(c\left(t_{2}\right)\right)
\end{array}\right|=0
$$

$i=3, \cdots, h d+1$.

We may assume that $c_{g}=c_{0}$ lies in $f_{0}$ (by choosing appropriate basis $f_{0}, f_{1}, f_{2}$ of $\mathbb{L})$. By (2.29), the Zariski tangent space $T_{c_{0}} P\left(\Gamma_{\mathbb{L}}\right)$ is defined by equations

$$
\left|\begin{array}{lll}
f_{2}\left(c_{0}\left(t_{i}\right)\right) & f_{1}\left(c_{0}\left(t_{i}\right)\right) & \frac{\partial f_{0}\left(c_{0}\left(t_{i}\right)\right)}{\partial \alpha} \\
f_{2}\left(c_{0}\left(t_{1}\right)\right) & f_{1}\left(c_{0}\left(t_{1}\right)\right) & \frac{\left.\partial f_{0}\left(t_{0}\right)\right)}{\partial \alpha} \\
f_{2}\left(c_{0}\left(t_{2}\right)\right) & f_{1}\left(c_{0}\left(t_{2}\right)\right) & \frac{\partial f_{0}\left(c_{0}\left(t_{2}\right)\right)}{\partial \alpha}
\end{array}\right|=0
$$

where $i=3, \cdots, h d+1, \alpha \in T_{c_{0}} P\left(\Gamma_{\mathbb{L}}\right)$. This is equivalent to that the column vectors of

$$
\left(\begin{array}{ccc}
f_{2}\left(c_{0}\left(t_{h d+1}\right)\right. & f_{1}\left(c_{0}\left(t_{h d+1}\right)\right) & \frac{\partial f_{0}\left(c_{0}\left(t_{h d+1}\right)\right)}{\partial \alpha_{0}} \\
\vdots & \vdots & \vdots \\
f_{2}\left(c_{0}\left(t_{2}\right)\right) & f_{1}\left(c_{0}\left(t_{2}\right)\right) & \frac{\partial f_{0}\left(c_{0}\left(t_{2}\right)\right)}{\partial \alpha_{0}} \\
f_{2}\left(c_{0}\left(t_{1}\right)\right) & f_{1}\left(c_{0}\left(t_{1}\right)\right) & \frac{\partial f_{0}\left(c_{0}\left(t_{1}\right)\right)}{\partial \alpha_{0}}
\end{array}\right)
$$

are linearly dependent, for some $\alpha_{0} \in T_{c_{0}} P\left(\Gamma_{\mathbb{L}}\right)$. Since

$$
\left(\begin{array}{c}
f_{2}\left(c_{0}\left(t_{h d+1}\right)\right. \\
\vdots \\
f_{2}\left(c_{0}\left(t_{2}\right)\right) \\
f_{2}\left(c_{0}\left(t_{1}\right)\right)
\end{array}\right),\left(\begin{array}{c}
f_{1}\left(c_{0}\left(t_{h d+1}\right)\right. \\
\vdots \\
f_{1}\left(c_{0}\left(t_{2}\right)\right) \\
f_{1}\left(c_{0}\left(t_{1}\right)\right)
\end{array}\right)
$$

are linearly independent, there are complex numbers $\epsilon_{i}, i=1,2$ such that

$$
\left(\begin{array}{c}
\frac{\partial f_{0}\left(c_{0}\left(t_{h d+1}\right)\right)}{\partial \alpha_{0}} \\
\vdots \\
\frac{\partial f_{0}\left(c_{0}\left(t_{2}\right)\right)}{\partial \alpha_{0}} \\
\frac{\partial f_{0}\left(c_{0}\left(t_{1}\right)\right)}{\partial \alpha_{0}}
\end{array}\right)=\epsilon_{2}\left(\begin{array}{c}
f_{2}\left(c_{0}\left(t_{h d+1}\right)\right. \\
\vdots \\
f_{2}\left(c_{0}\left(t_{2}\right)\right) \\
f_{2}\left(c_{0}\left(t_{1}\right)\right)
\end{array}\right)+\epsilon_{1}\left(\begin{array}{c}
f_{1}\left(c_{0}\left(t_{h d+1}\right)\right. \\
\vdots \\
f_{1}\left(c_{0}\left(t_{2}\right)\right) \\
f_{1}\left(c_{0}\left(t_{1}\right)\right)
\end{array}\right)
$$

Let $f_{3}=\sum_{i=1}^{2} \epsilon_{i} f_{i}$ where $\epsilon_{i}$ are fixed. We may assume that $\mathbb{L}_{1}$ is the pencil containing $f_{0}, f_{3}$. The equation (2.33) becomes

$$
\frac{\partial f_{0}\left(c_{0}(t)\right)}{\partial \alpha_{0}}-f_{3}\left(c_{0}(t)\right)=0, \text { for all } t \in \mathbf{P}^{1} .
$$


Then just as in the (2.25),

$$
T_{c_{0}} \Gamma_{\mathbb{L}} \simeq T_{c_{0}} \Gamma_{f_{0}} \oplus \mathbb{C}<\overrightarrow{f_{1}}>_{M} \oplus \mathbb{C}<\overrightarrow{f_{2}}>_{M} .
$$

and

$$
T_{c_{0}} \Gamma_{\mathbb{L}_{1}} \simeq T_{c_{0}} \Gamma_{f_{0}} \oplus \mathbb{C}<\overrightarrow{f_{3}}>_{M} .
$$

Then the lemma follows.

प

\subsubsection{Space of rational curves, $M$}

The main purpose of this section is to introduce analytic coordinates of a neighborhood of $M$, which will be used as local coordinates of the blow-up $\tilde{M}$ of $M$ in the computation of a Jacobian matrix (2.87). These coordinates identify a neighborhood of $\tilde{M}$ outside of exceptional divisor with a neigborhood of $M$. They are crucial.They are crucial.

Let $\tilde{c}_{2}=\left(\tilde{c}_{2}^{0}(t), \cdots, \tilde{c}_{2}^{n}\right) \in M$ with

$$
\tilde{c}_{2}^{i} \neq 0 \in H^{0}\left(\mathcal{O}_{\mathbf{P}^{1}}(d)\right), i=0, \cdots, n .
$$

We assume $\tilde{c}_{2}^{i}(t)=0, i \leq n-2$, have $(n-1) d$ distinct zeros

$$
\tilde{\theta}_{i}^{j}, \text { for } i \leq n-2 \text {. }
$$

Then the first $n-1$ components, $H^{0}\left(\mathcal{O}_{\mathbf{P}^{1}}(d)\right)$ of

$$
M=H^{0}\left(\mathcal{O}_{\mathbf{P}^{1}}(d)\right)^{\oplus n+1}
$$

has local analytic coordinates

$$
r_{l}, \theta_{i}^{j}, j=1, \cdots, d, l=0, \cdots, n-2, i=0, \cdots, n-2
$$

with $r_{i} \neq 0$ around $\tilde{c}_{2}^{i}$ such that

$$
c^{i}(t)=r_{i} \prod_{j=1}^{d}\left(t-\theta_{i}^{j}\right),
$$

and last two components, $H^{0}\left(\mathcal{O}_{\mathbf{P}^{1}}(d)\right)$ of

$$
M=H^{0}\left(\mathcal{O}_{\mathbf{P}^{1}}(d)\right)^{\oplus n+1}
$$

have affine coordinates $r_{i}, \theta_{i}^{j}, i=n-1, n$ near $\tilde{c}_{2}$,

$$
r_{n-1}=c_{n-1}^{0}, r_{n}=c_{n}^{0}, \theta_{i}^{j}=\frac{c_{i}^{j}}{c_{i}^{0}}, \text { for } i=n-1, n, j=1, \cdots, d
$$

where $c_{i}^{j}$ are coefficients of $c^{i}(t)$. Assume the values of coordinates

$$
c_{i}^{0}, i=n-1, n
$$


for the point $\tilde{c}_{2}$ are non-zeros, i.e. $\tilde{c}_{2}$ lies in the neighborhood of this coordinate system:

$$
r_{l}, \theta_{i}^{j}, \text { for } 0 \leq l, i \leq n, j=1, \cdots, d, r_{l} \neq 0 .
$$

Let a part of coordinates values for $\tilde{c}_{2}$ be

$$
\begin{gathered}
r_{l}=y_{l}, \theta_{i}^{j}=\tilde{\theta}_{i}^{j}, l=0, \cdots, n-2, i=0, \cdots, n, j=1, \cdots, d \\
c_{n-1}^{0}=y_{n-1}, c_{n}^{0}=y_{n} .
\end{gathered}
$$

Let $q$ be a generic, homogeneous quadratic polynomial in $z_{0}, \cdots, z_{n}$. Let

$$
h(c, t)=\delta_{1} q(c(t))+\delta_{2} c_{n-1}(t) c_{n}(t) .
$$

for $c \in M$, where $\delta_{i}, i=1,2$ are two none zero complex numbers. Let $\beta_{1}, \cdots, \beta_{2 d}$ be the zeros of $h\left(\tilde{c}_{2}, t\right)=0$. Furthermore we assume $\beta_{i}, i=1, \cdots, 2 d$ are distinct and non-zeros for generic $\delta_{i}$ and specific $q=z_{1} \cdots z_{n-2}$.

Proposition 2.10. Let $U_{\tilde{c}_{2}} \subset M$ be an analytic neighborhood of $\tilde{c}_{2}$.

Let

$$
g: U_{\tilde{c}_{2}} \rightarrow \mathbb{C}^{(n+1)(d+1)}
$$

be a regular map that is defined by

$$
\begin{gathered}
g\left(\theta_{0}^{1}, \cdots, \theta_{n}^{d}, r_{0}, \cdots, r_{n}\right) \\
\| \\
\left(\theta_{0}^{1}, \cdots, \theta_{n-2}^{d}, h\left(c, \beta_{1}\right), \cdots, h\left(c, \beta_{2 d}\right), r_{0}, \cdots, r_{n}\right) .
\end{gathered}
$$

Then $g$ is an isomorphism to its image.

Proof. It suffices to prove the differential of $g$ at $\tilde{c}_{2}$ is an isomorphism for a SPECIFIC $q$. So we assume that

$$
\delta_{1}=\delta_{2}=1, q=z_{1} \cdots z_{n-2}
$$

This is a straightforward calculation of the Jacobian of $g$. We may still assume that $\beta_{i}, i=1, \cdots, 2 d$ are distinct. The Jacobian

$$
\frac{\partial g\left(\tilde{\theta}_{0}^{1}, \cdots, \tilde{\theta}_{n-2}^{d}, y_{0}, \cdots, y_{n}, h\left(\tilde{c}_{2}, \beta_{1}\right), \cdots, h\left(\tilde{c}_{2}, \beta_{2 d}\right)\right.}{\partial\left(\theta_{0}^{1}, \cdots, \theta_{n-2}^{d}, r_{0}, \cdots, r_{n}, \theta_{n-1}^{1}, \cdots, \theta_{n}^{d}\right)}
$$

is equal to another Jacobian

$$
J a=\left|\begin{array}{ccc}
\frac{\partial h\left(c, \beta_{1}\right)}{\partial \theta_{n-1}^{1}} & \cdots & \frac{\partial h\left(c, \beta_{1}\right)}{\partial \theta_{n}^{d}} \\
\vdots & \vdots & \vdots \\
\frac{\partial h\left(c, \beta_{2 d}\right)}{\partial \theta_{n-1}^{1}} & \cdots & \frac{\partial h\left(c, \beta_{2 d}\right)}{\partial \theta_{n}^{d}}
\end{array}\right|_{\tilde{c}_{2}}
$$

Let $T_{i}, i=0, d$ be the determinant

$$
\left|\begin{array}{ccc}
\beta_{i+1} & \cdots & \beta_{i+1}^{d} \\
\vdots & \vdots & \vdots \\
\beta_{i+d} & \cdots & \beta_{i+d}^{d}
\end{array}\right|
$$


Then we compute the determinant to have

$$
J a=(-1)^{d} \delta_{2} T_{0} T_{d} \prod_{i=1}^{d}\left(\tilde{c}_{2}^{n-1}\left(\beta_{d+i}\right) \tilde{c}_{2}^{n}\left(\beta_{i}\right)-\tilde{c}_{2}^{n-1}\left(\beta_{i}\right) \tilde{c}_{2}^{n}\left(\beta_{d+i}\right)\right) .
$$

Since $\beta_{i}$ are distinct and non-zeros,

$$
T_{0} \neq 0, T_{d} \neq 0 .
$$

Since $\frac{\tilde{c}_{2}^{n-1}(t)}{\tilde{c}_{2}^{n}(t)}$ is a rational function and

$$
\operatorname{deg}\left(\tilde{c}_{2}^{n-1}(t)\right)=\operatorname{deg}\left(\tilde{c}_{2}^{n}(t)\right)=d
$$

then we can always arrange the subscripts $i$ of $\beta_{i}$ such that each number

$$
\left(\frac{\tilde{c}_{2}^{n-1}\left(\beta_{d+i}\right)}{\tilde{c}_{2}^{n}\left(\beta_{d+i}\right)}-\frac{\tilde{c}_{2}^{n-1}\left(\beta_{i}\right)}{\tilde{c}_{2}^{n}\left(\beta_{i}\right)}\right)
$$

is not zero. Hence

$$
\prod_{i=1}^{d}\left(\tilde{c}_{2}^{n-1}\left(\beta_{d+i}\right) \tilde{c}_{2}^{n}\left(\beta_{i}\right)-\tilde{c}_{2}^{n-1}\left(\beta_{i}\right) \tilde{c}_{2}^{n}\left(\beta_{d+i}\right)\right) \neq 0
$$

Thus $J a$ is non-zero.

Therefore

$$
\frac{\partial g\left(\tilde{\theta}_{0}^{1}, \cdots, \tilde{\theta}_{n-2}^{d}, y_{0}, \cdots, y_{n}, h\left(\tilde{c}_{2}, \beta_{1}\right), \cdots, h\left(\tilde{c}_{2}, \beta_{2 d}\right)\right)}{\partial\left(\theta_{0}^{1}, \cdots, \theta_{n-2}^{d}, r_{0}, \cdots, r_{n}, \theta_{n-1}^{1}, \cdots, \theta_{n}^{d}\right)} \neq 0
$$

We complete the proof.

$\square$

Definition 2.11. Let $\epsilon_{i}=h\left(c, \beta_{i}\right), i=1, \cdots, 2 d$. Then by proposition 2.10,

$$
\theta_{0}^{1}, \cdots, \theta_{n-2}^{d}, r_{0}, \cdots, r_{n}, \epsilon_{1}, \cdots, \epsilon_{2 d}
$$

are the local analytic coordinates of $M$ around $\tilde{c}_{2}$, and $\tilde{c}_{2}$ corresponds to the coordinate values

$$
\begin{gathered}
\theta_{i}^{j}=\tilde{\theta}_{i}^{j}, i \leq n-2, j=1, \cdots, d \\
r_{l}=y_{l} \neq 0, l=0, \cdots, n \\
\epsilon_{i}=0, i=1, \cdots, 2 d
\end{gathered}
$$

\subsubsection{Differential sheaf}

Next we prove theorem 1.1, i.e.

$$
H^{1}\left(N_{c_{0} / X_{0}}\right)=0
$$

at generic $\left(c_{0},\left[f_{0}\right]\right) \in \Gamma$ for Calabi-Yau $X_{0}$. 
Choose a homogeneous coordinate system $\left[z_{0}, \cdots, z_{n}\right]$ for $\mathbf{P}^{n}$. Let

$$
f_{3}=z_{0} \cdots z_{n-2}\left(\delta_{1} q+\delta_{2} z_{n-1} z_{n}\right) .
$$

where $\delta_{i}$ are two complex non-zero complex numbers, and $q$ is a generic quadric in $\mathbf{P}^{n}$. Let $\tilde{c}_{2} \in M$ and

$$
f_{3}\left(\tilde{c}_{2}(t)\right) \neq 0 .
$$

As before we denote the zeros of $\tilde{c}_{2}^{i}(t)=0$ by $\tilde{\theta}_{i}^{j}, j \leq n-2$ and zeros of

$$
\left(\delta_{1} q+\left.\delta_{2} z_{n-1} z_{n}\right|_{\tilde{c}_{2}(t)}\right)=0
$$

by $\beta_{i}, i=1, \cdots, 2 d$. We assume $\tilde{\theta}_{i}^{j}, j \leq n-2$ are distinct, and $\beta_{i}, i=1, \cdots, 2 d$ are also distinct.

Lemma 2.12. Let $t_{1}, \cdots, t_{h d}$ be the zeros of $f_{3}\left(\tilde{c}_{2}(t)\right)$. Recall in definition 2.11,

$$
\begin{gathered}
\theta_{i}^{j}=\tilde{\theta}_{i}^{j}, i \leq n-2, j=1, \cdots, d \\
r_{l}=y_{l} \neq 0, l=0, \cdots, n \\
\epsilon_{i}=0, i=1, \cdots, 2 d
\end{gathered}
$$

are analytic coordinates of $M$ around the point $\tilde{c}_{2}$.

Then the Jacobian matrix

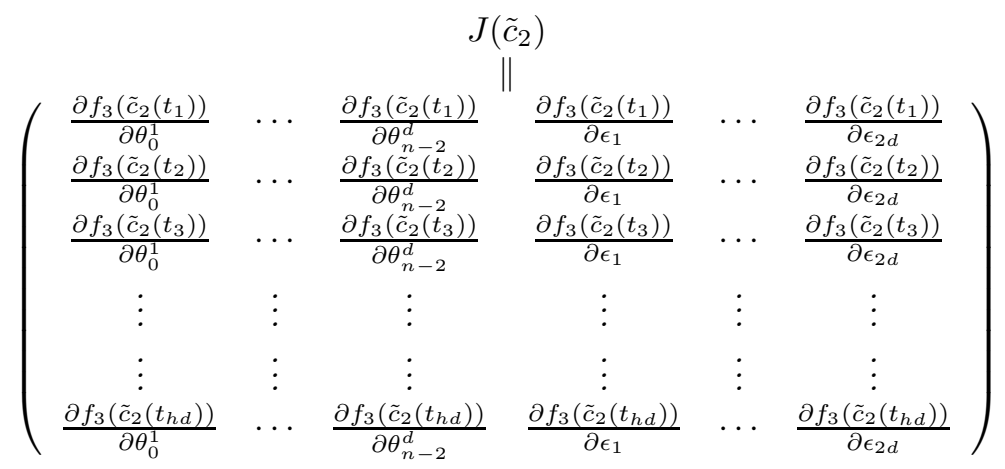

is equal to a diagonal matrix $D$ whose diagonal entries are

$$
\begin{gathered}
\frac{\partial f_{3}\left(\tilde{c}_{2}\left(t_{1}\right)\right)}{\partial \theta_{0}^{1}}, \cdots, \frac{\partial f_{3}\left(\tilde{c}_{2}\left(t_{(n-1) d}\right)\right)}{\partial \theta_{n-2}^{d}}, \\
\frac{\partial f_{3}\left(\tilde{c}_{2}\left(t_{(n-1) d+1}\right)\right)}{\partial \epsilon_{1}}, \cdots, \frac{\partial f_{3}\left(\tilde{c}_{2}\left(t_{h d}\right)\right)}{\partial \epsilon_{2 d}} .
\end{gathered}
$$

which are all non-zeros.

Proof. Note $\tilde{\theta}_{i}^{j}$ are distinct and $\beta_{i}$ are also distinct by the genericity of $q$. Thus the coordinates in definition 2.11 exist. It suffices to show all non diagonal entries of (2.53) are zeros. We can rewrite

$$
f_{3}(c(t))=y \prod_{j=1}^{h d}\left(t-\alpha_{j}\right)
$$


around $\tilde{c}_{2}$. Then $y, \alpha_{j}$ are all functions of the analytic coordinates in definition 11,

$$
\theta_{i}^{j}, \epsilon_{1}, \cdots, \epsilon_{2 d}, r_{l} \text {. }
$$

(Next we simply show that $\alpha_{i}$ correspond to coordinates $\theta_{i}^{j}, \epsilon_{n}, i \leq n-2$ ). By the definition the Jacobian matrix of

$$
\frac{\partial\left(\alpha_{1}, \cdots, \alpha_{h d}\right)}{\partial\left(\theta_{0}^{1}, \cdots, \theta_{n-2}^{d}, \epsilon_{1}, \cdots, \epsilon_{2 d}\right)}
$$

is a non-zero diagonal matrix when evaluated at $\tilde{c}_{2}$. Since it is clear that

$$
\frac{\partial f_{3}\left(\tilde{c}_{2}\left(t_{i}\right)\right)}{\partial \alpha_{j}}=0, i \neq j
$$

the non-diagonals of $J\left(\tilde{c}_{2}\right)$ are also 0 . Upto a non-zero constant, the diagonal entries $J\left(\tilde{c}_{2}\right)$ are

$$
\prod_{j \neq 1}\left(t_{1}-t_{j}\right), \prod_{j \neq 2}\left(t_{2}-t_{j}\right), \cdots, \prod_{j \neq h d}\left(t_{h d}-t_{j}\right) .
$$

We complete the proof. $\square$

\subsubsection{Non-vanishing $h d-1$-form $\omega$}

The section 2.1.4 and lemma 2.12 are just the preparation for the proof. Section 2.1.3 is the one that is meant to dig into the problem, but it is only to the first order. With only the results in section 2.1.3, we can't go far because theorem 1.1 touches upon the higher orders of deformations of pairs. The following lemma is the reflection of this philosophy.

LEMMA 2.13.

The hd-1 form $\omega$ defined in (1.5) is a non-zero form when it is evaluated at generic points of $P\left(\Gamma_{\mathbb{L}}\right)$, i.e. the reduction $\bar{\omega}$ in the module,

$$
H^{0}\left(\Omega_{M} \otimes \mathcal{O}_{P\left(\Gamma_{\mathbb{L}}\right)}\right)
$$

is non zero.

This is the proposition 1.2.

It suffices to prove lemma 2.13 for a special choice of $f_{0}, f_{1}, f_{2}$, and we only need to produce one point (any point) on $P\left(\Gamma_{\mathbb{L}}\right)$, at which $\omega$ is non-vanishing.

So let $z_{0}, z_{1}, \cdots, z_{n}$ be general homogeneous coordinates of $\mathbf{P}^{n}$. Let

$$
f_{2}=z_{0} \cdots z_{n}
$$

Let

$$
f_{1}=z_{0} \cdots z_{n-2} q,
$$

where $q$ is a generic quadratic polynomial in $z_{0}, \cdots, z_{n}$. Choose another generic $f_{0}$. We obtain an open set $\mathbb{L}_{1}$ of pencil through $f_{0}, f_{2}$, and an open set $\mathbb{L}$ of 2 -dimensional 
plane containing $f_{0}, f_{1}, f_{2}$ in $S$. Let $P\left(\Gamma_{\mathbb{L}}\right)$ and $P\left(\Gamma_{\mathbb{L}_{1}}\right)$ be as defined in lemmas 2.8, 2.9. We choose $P\left(\Gamma_{\mathbb{L}_{1}}\right)$ to be irreducible, and to be contained in $P\left(\Gamma_{\mathbb{L}}\right)$ for generic $f_{0}$. We may assume a generic point $c=\left(c^{0}, \cdots, c^{n}\right) \in P\left(\Gamma_{\mathbb{L}_{1}}\right)$ does not have multiple zeros with coordinates planes, i.e. $c^{i}=0, i=0, \cdots$ have $h d$ distinct roots (This is because we can always choose a generic coordinates' system $z_{i}$ with respect to the fixed $\left.c_{0}\right)$. Let

$$
c_{2} \in P\left(\Gamma_{\mathbb{L}_{1}}\right) \subset P\left(\Gamma_{\mathbb{L}}\right)
$$

such that $f_{2}\left(c_{2}(t)\right)=0$. By the genericity of $f_{0}$ and $z_{0}$, we assume

$$
P\left(\Gamma_{\mathbb{L}_{1}}\right) \not \subset\left\{z_{0}=0\right\} .
$$

Thus we may assume $c_{2}=\left[0, c_{2}^{1}, \cdots, c_{2}^{n}\right]$ where $c_{2}^{i}, i \neq 0$ are non-zero sections of $H^{0}\left(\mathcal{O}_{\mathbf{P}^{1}}(d)\right)$. Since the proofs for other cases with more zero sections $c_{2}^{i}$ are the same, it suffices for us to prove this case only. It is not difficult to see $\omega$ is zero at $c_{2}$ for the choice of $\mathbb{L}$. But we would like to show that $\omega$ is not identically zero on $P\left(\Gamma_{\mathbb{L}}\right)$. The technique is "blow-up".

Let's first describe it in the most general term: we would construct a birational map, the composition of successive blow-ups,

$$
\pi: Y \rightarrow P\left(\Gamma_{\mathbb{L}}\right)
$$

which is an isomorphism on $Y-\pi^{-1}\left(B^{\prime}\right)$ where $B^{\prime}$ is a proper closed sub-scheme of $P\left(\Gamma_{\mathbb{L}}\right)$ that contains $c_{2}$, and $\pi^{-1}\left(B^{\prime}\right)$ is also a proper closed sub-scheme. We then compute to find out that

$$
\pi^{*}(\omega)=g \omega^{\prime}
$$

on $Y$ where $g$ is a non-zero rational function on $Y$, and $\omega^{\prime}$ does not vanish at a point $p \in \pi^{-1}\left(B^{\prime}\right)$. Because $\pi$ is birational, $\omega$ is a non-zero form restricted to $P\left(\Gamma_{\mathbb{L}}\right)$. The key to this assertion is that $\omega^{\prime}$ is non-zero at one point $p$. The process of blow-ups is messy and lengthy. In order to organize them, we divide them into two different types:

(1) blow-ups used to resolve the vanishing of sections $c_{2}^{i}, i=0, \cdots, n$ in the rational map $c_{2}$. These will be only used in step 1 below.

(2) blow-ups used to resolve the multiple zeros of $\tilde{c}_{2}$ with coordinates planes. This is the case where $c_{2}$ could be a constant map. These will be used in step 2 below.

Let's see the details. Let

$$
B_{1} \subset \mathbb{C}^{(n+1)(d+1)}
$$

be the subvariety that is equal to

$$
\{0\} \oplus H^{0}\left(\mathcal{O}_{\mathbf{P}^{1}}(d)\right) \oplus \cdots \oplus H^{0}\left(\mathcal{O}_{\mathbf{P}^{1}}(d)\right) .
$$

Let

$$
\tilde{\mathbb{C}}^{(n+1)(d+1)} \stackrel{\pi_{1}}{\rightarrow} \quad \mathbb{C}^{(n+1)(d+1)}
$$

be the blow-up of $\mathbb{C}^{(n+1)(d+1)}$ along $B_{1}$. It is clear that

$$
\begin{gathered}
\tilde{\mathbb{C}}^{(n+1)(d+1)} \\
\| \\
\tilde{\mathbb{C}}^{d+1} \times H^{0}\left(\mathcal{O}_{\mathbf{P}^{1}}(d)\right) \times \cdots \times H^{0}\left(\mathcal{O}_{\mathbf{P}^{1}}(d)\right)
\end{gathered}
$$


where $\tilde{\mathbb{C}}^{d+1}$ is the blow-up of $\mathbb{C}^{d+1}$ at the origin. Now let $\tilde{M}$ and $\tilde{P}\left(\Gamma_{\mathbb{L}}\right)\left(\tilde{P}\left(\Gamma_{\mathbb{L}_{1}}\right)\right)$ be the strict transforms of $M$ and $P\left(\Gamma_{\mathbb{L}}\right)\left(P\left(\Gamma_{\mathbb{L}_{1}}\right)\right)$ respectively. Let $E_{1}, E_{1 \mathbb{L}}$ be their exceptional divisors. Let $\tilde{c}_{2} \in \tilde{P}\left(\Gamma_{\mathbb{L}_{1}}\right)$ be an inverse of $c_{2}$ under the map

$$
\tilde{P}\left(\Gamma_{\mathbb{L}_{1}} \stackrel{\left.\pi_{1}\right|_{\tilde{P}\left(\Gamma_{\mathbb{L}_{1}}\right)}}{\rightarrow} P\left(\Gamma_{\mathbb{L}_{1}}\right)\right.
$$

(such $\tilde{c}_{2}$ is independent of choice of $q$ ).

Lemma 2.14. Let $\left(t_{1}, \cdots, t_{h d}\right) \in \operatorname{Sym}^{h d}\left(\mathbf{P}^{1}\right)$ be generic. Let

$$
\begin{gathered}
\psi_{i}=\left|\begin{array}{ll}
f_{0}\left(c\left(t_{1}\right)\right) & f_{2}\left(c\left(t_{1}\right)\right) \\
f_{0}\left(c\left(t_{2}\right)\right) & f_{2}\left(c\left(t_{2}\right)\right)
\end{array}\right| d f_{1}\left(c\left(t_{i}\right)\right)+\left|\begin{array}{ll}
f_{2}\left(c\left(t_{1}\right)\right) & f_{1}\left(c\left(t_{1}\right)\right) \\
f_{2}\left(c\left(t_{2}\right)\right) & f_{1}\left(c\left(t_{2}\right)\right)
\end{array}\right| d f_{0}\left(c\left(t_{i}\right)\right) \\
+\left|\begin{array}{lll}
f_{1}\left(c\left(t_{1}\right)\right) & f_{0}\left(c\left(t_{1}\right)\right) \\
f_{1}\left(c\left(t_{2}\right)\right) & f_{0}\left(c\left(t_{2}\right)\right)
\end{array}\right| d f_{2}\left(c\left(t_{i}\right)\right)
\end{gathered}
$$

for $i=3,4, \cdots, h d+1$. Then vectors

$$
\begin{gathered}
\pi_{1}^{*}\left(\psi_{i}\right), i=3, \cdots, h d+1 \\
\pi_{1}^{*}\left(d f_{0}\left(c\left(t_{1}\right)\right)\right), \pi_{1}^{*}\left(d f_{0}\left(c\left(t_{2}\right)\right)\right), \\
\pi_{1}^{*}\left(d f_{1}\left(c\left(t_{1}\right)\right)\right), \pi_{1}^{*}\left(d f_{1}\left(c\left(t_{2}\right)\right)\right), \\
\pi_{1}^{*}\left(d f_{2}\left(c\left(t_{1}\right)\right)\right), \pi_{1}^{*}\left(d f_{2}\left(c\left(t_{2}\right)\right)\right)
\end{gathered}
$$

are linearly independent in the vector space $\left(T_{\tilde{c}} \tilde{M}\right)^{*}$, i.e. they form a basis, where $\tilde{c}$ lies in a non-empty open set of $\tilde{P}\left(\Gamma_{\mathbb{L}}\right)$, and $\tilde{c} \neq \tilde{c}_{2}$. 5

Proof. of lemma 2.14: The proof is long. Thus we divide it into two steps. It suffices to prove it for special $t_{1}, \cdots, t_{h d+1}$. First let $\tilde{c}_{2}$ be decomposed (according to (2.61)) to

$$
\tilde{c}_{2}=\left(\tilde{c}_{2}^{0}(t), \cdots, \tilde{c}_{2}^{n}(t)\right)
$$

where

$$
\tilde{c}_{2}^{0}(t) \in \mathbf{P}\left(H^{0}\left(\mathcal{O}_{\mathbf{P}^{1}}(d)\right), \tilde{c}_{2}^{i}(t) \in H^{0}\left(\mathcal{O}_{\mathbf{P}^{1}}(d)\right), i \neq 0 .\right.
$$

and the zeros of all $\tilde{c}_{2}^{i}, i=0, \cdots, n$ are $\tilde{\theta}_{i}^{j}$.

Step 1: Suppose that

$$
\tilde{\theta}_{i}^{j}, i=0, \cdots, n, j=1, \cdots, d,
$$

are distinct. In this case, we let $t_{1}, t_{2}$ be two points on $\mathbf{P}^{1}$ satisfying

$$
\left|\begin{array}{ll}
q \tilde{c}_{c_{2}\left(t_{1}\right)} & \tilde{c}_{2}^{n-1}\left(t_{1}\right) \tilde{c}_{2}^{n}\left(t_{1}\right) \\
\left.q\right|_{\tilde{c}_{2}\left(t_{2}\right)} & \tilde{c}_{2}^{n-1}\left(t_{2}\right) \tilde{c}_{2}^{n}\left(t_{2}\right)
\end{array}\right|=0 .
$$

\footnotetext{
5 The vectors in lemma 2.14 come from the expansion of $\phi_{i}$ :

$$
\begin{aligned}
\phi_{i}= & \left|\begin{array}{ll}
f_{0}\left(c\left(t_{1}\right)\right) & f_{2}\left(c\left(t_{1}\right)\right) \\
f_{0}\left(c\left(t_{2}\right)\right) & f_{2}\left(c\left(t_{2}\right)\right)
\end{array}\right| d f_{1}\left(c\left(t_{i}\right)\right)+\left|\begin{array}{ll}
f_{2}\left(c\left(t_{1}\right)\right) & f_{1}\left(c\left(t_{1}\right)\right) \\
f_{2}\left(c\left(t_{2}\right)\right) & f_{1}\left(c\left(t_{2}\right)\right)
\end{array}\right| d f_{0}\left(c\left(t_{i}\right)\right) \\
& +\left|\begin{array}{ll}
f_{1}\left(c\left(t_{1}\right)\right) & f_{0}\left(c\left(t_{1}\right)\right) \\
f_{1}\left(c\left(t_{2}\right)\right) & f_{0}\left(c\left(t_{2}\right)\right)
\end{array}\right| d f_{2}\left(c\left(t_{i}\right)\right)+\sum_{l=0, j=1}^{l=2, j=2} h_{l j}^{i}(c) d f_{l}\left(c\left(t_{j}\right)\right)
\end{aligned}
$$
}

where $h_{l j}^{i}$ are polynomials in $c$. 
Let

$$
f_{3}=z_{0} \cdots z_{n-2}\left(\delta_{1} q+\delta_{2} z_{n-1} z_{n}\right)
$$

where

$$
\delta_{1}=\left|\begin{array}{ll}
f_{0}\left(c_{2}\left(t_{1}\right)\right) & f_{2}\left(\tilde{c}_{2}\left(t_{1}\right)\right) \\
f_{0}\left(c_{2}\left(t_{2}\right)\right) & f_{2}\left(\tilde{c}_{2}\left(t_{2}\right)\right)
\end{array}\right|, \quad \delta_{2}=\left|\begin{array}{ll}
f_{1}\left(\tilde{c}_{2}\left(t_{1}\right)\right) & f_{0}\left(c_{2}\left(t_{1}\right)\right) \\
f_{1}\left(\tilde{c}_{2}\left(t_{2}\right)\right) & f_{0}\left(c_{2}\left(t_{2}\right)\right)
\end{array}\right| .
$$

Then let $t_{3}, \cdots, t_{h d}$ be zeros of

$$
\begin{gathered}
f_{3}\left(\left(\tilde{c}_{2}(t)\right)\right. \\
\| \\
\tilde{c}_{2}^{0}(t) \cdots \tilde{c}_{2}^{n-2}(t)\left(\left.\delta_{1} q\right|_{\tilde{c}_{2}(t)}+\delta_{2} \tilde{c}_{2}^{n-1}(t) \tilde{c}_{2}^{n}(t)\right) \\
\| \\
0,
\end{gathered}
$$

other than $\tilde{\theta}_{0}^{1}, \tilde{\theta}_{1}^{1}$. Let $t_{h d+1}$ be generic. Because $f_{0}, q$ are generic, the zeros of

$$
\left.\delta_{1} q\right|_{\tilde{c}_{2}(t)}+\delta_{2} \tilde{c}_{2}^{n-1}(t) \tilde{c}_{2}^{n}(t)
$$

are distinct and non-zeros. Thus

$$
t_{1}, t_{2}, t_{3}, \cdots, t_{h d+1}
$$

are distinct. Let's set-up the coordinates of $\tilde{\mathbb{C}}^{(n+1)(d+1)}$. Let $c_{i}^{j}, i=0, \cdots, n, j=$ $0, \cdots, d$ be the coefficients of $n+1$ tuples of sections of $H^{0}\left(\mathcal{O}_{\mathbf{P}^{1}}(d)\right)$. They are the affine coordinates of

$$
\mathbb{C}^{(n+1)(d+1)} .
$$

Each section of $\mathcal{O}_{\mathbf{P}^{1}}(d)$ in an analytic neighborhood excluding those with multiple zeros can be written as

$$
c_{i}(t)=r_{i} \Pi_{j=1}^{d}\left(t-\theta_{i}^{j}\right) .
$$

for $i \leq n-2, r_{i} \neq 0$, and

$$
c_{i}(t)=r_{i} \Pi_{j=1}^{d}\left(t-\mu_{i}^{j}\right)
$$

for $i=n-1, n$ and $r_{i} \neq 0$.

Then $r_{m}, \mu_{i}^{j}, \theta_{i}^{j}$ are local analytic coordinates for an analytic open set $U_{\widetilde{\mathbb{C}}^{(n+1)(d+1)}}$ of the blow-up

$$
\tilde{\mathbb{C}}^{(n+1)(d+1)}
$$

centered around $\tilde{c}_{2} \in \tilde{\mathbb{C}}^{(n+1)(d+1)}$ (now $r_{m}$ as coordinates of $\tilde{\mathbb{C}}^{(n+1)(d+1)}$ could be zeros). We may assume $\tilde{c}_{2}$ lies in the neighborhood of the coordinates and $\tilde{c}_{2}$ has specific coordinates

$$
\begin{gathered}
r_{0}=0 \\
\theta_{i}^{j}=\tilde{\theta}_{i}^{j}, i=0, \cdots, n-2, j=1, \cdots, d, \\
\mu_{i}^{j}=\tilde{\theta}_{i}^{j}, i=n-1, n, j=1, \cdots, d, \\
r_{j}=y_{j} \neq 0, j=1, \cdots, n
\end{gathered}
$$

\footnotetext{
${ }^{6}$ If not, we continue to have successive blow-ups till the pre-image of $c_{2}$ lies in the coordinates' neighborhood.
} 
Because $t_{3}, \cdots, t_{h d}$ are distinct and the last $2 d$ of them are non-zeros, by the definition 2.11, in this neighborhood we have another analytic coordinates

$$
\theta_{0}^{1}, \cdots, \theta_{n-2}^{d}, r_{0}, \cdots, r_{n}, \epsilon_{1}, \cdots, \epsilon_{2 d}
$$

To see this, we notice that there is an isomorphism $\widetilde{\mathbb{C}}^{h(d+1)} \rightarrow \mathbb{C}^{h(d+1)}$ outside of $B_{1}$. Thus above the coordinates (2.76) from definition 2.11 are also local coordinates of $\tilde{\mathbb{C}}^{h(d+1)}$. In the rest of calculation we use these coordinates for $\tilde{\mathbb{C}}^{h(d+1)}$. Then the finite set

$$
\mathcal{B}=\left\{d r_{l}, d \theta_{i}^{j}, d \epsilon_{m}\right\}_{l=0, \cdots, n, i \leq n-2, j=1, \cdots, d, m=1, \cdots, 2 d}
$$

is a basis for $\left(T_{\tilde{c}} U_{\tilde{\mathbb{C}}^{(n+1)(d+1)}}\right)^{*}$ where $\tilde{c} \in U_{\tilde{\mathbb{C}}^{(n+1)(d+1)}}$.

For this step, it is clear that lemma 2.14 follows from

LEMMA 2.15. Let $\mathcal{A}^{\prime}$ be the coefficient matrix of hd+5 vectors in (2.66) under the basis $\mathcal{B}$. Then $\mathcal{A}^{\prime}$ has full rank near $\tilde{c}_{2}$.

Proof. of lemma 2.15:

Set-up: Assume the blow-up in the lemma 2.15. The row vectors in $\mathcal{A}^{\prime}$ are vectors in (2.66). We place them from top to bottom in the following order

$$
\begin{gathered}
\pi_{1}\left(\psi_{3}\right), \\
\vdots \\
\pi_{1}^{*}\left(\psi_{h d}\right), \\
\pi_{1}^{*}\left(\psi_{h d+1}\right) \\
\pi_{1}^{*}\left(d f_{2}\left(c\left(t_{1}\right)\right)\right), \\
\pi_{1}^{*}\left(d f_{2}\left(c\left(t_{2}\right)\right)\right), \\
\pi_{1}^{*}\left(d f_{1}\left(c\left(t_{1}\right)\right)\right), \\
\pi_{1}^{*}\left(d f_{1}\left(c\left(t_{2}\right)\right)\right), \\
\pi_{1}^{*}\left(d f_{0}\left(c\left(t_{1}\right)\right)\right), \\
\pi_{1}^{*}\left(d f_{0}\left(c\left(t_{2}\right)\right)\right) .
\end{gathered}
$$

The basis vectors are listed, from left to right, as

$$
d \theta_{0}^{2}, \cdots, \widehat{d} \theta_{1}^{1}, \cdots, d \theta_{n-2}^{d}, d \epsilon_{1}, \cdots, d \epsilon_{2 d}, d r_{0}, d \theta_{0}^{1}, d \theta_{1}^{1}, d r_{1}, \cdots, d r_{n} .
$$

( $\widehat{\cdot}$ denotes omitting). So $\mathcal{A}^{\prime}$ is a matrix of size $[h d+5] \times[(n+1)(d+1)]$ where $n \geq 4$. So there are more columns than rows. Let $\mathcal{A}$ be the sub square matrix of $\mathcal{A}^{\prime}$ which is the coefficient matrix of vectors in (2.66) under the basis

$$
\begin{gathered}
d \theta_{0}^{2}, \cdots, \widehat{d} \theta_{1}^{1}, \cdots, d \theta_{n-2}^{d}, d \epsilon_{1}, \cdots, d \epsilon_{2 d}, \\
d r_{0}, d \theta_{0}^{1}, d \theta_{1}^{1}, d r_{1}, d r_{2}, d r_{n-1}, d r_{n} .
\end{gathered}
$$

This can be arranged because $n \geq 4$. Then matrix $\mathcal{A}$ is the matrix partitioned as

$$
\left(\begin{array}{ccc}
\mathcal{A}_{11} & \mathcal{A}_{12} & \mathcal{A}_{13} \\
\mathcal{A}_{21} & \mathcal{A}_{22} & \mathcal{A}_{23} \\
\mathcal{A}_{31} & \mathcal{A}_{32} & \mathcal{A}_{33} \\
\mathcal{A}_{41} & \mathcal{A}_{42} & \mathcal{A}_{43} \\
\mathcal{A}_{51} & \mathcal{A}_{52} & \mathcal{A}_{53}
\end{array}\right)
$$


where all $\mathcal{A}_{i j}$ are matrices of different sizes. We should describe them, one-by-one, as follows:

In the following $O(1)$ denotes a polynomial function on $U_{\tilde{\mathbb{C}}^{(n+1)(d+1)}}$ of forms

$$
\left(t_{l}-\theta_{i}^{j}\right) O, \epsilon_{i} O
$$

where $O$ are polynomial functions on $U_{\widetilde{\mathbb{C}}^{(n+1)(d+1)}}$. Note that by the definition

$$
t_{l}=\tilde{\theta}_{i}^{j}, i \leq n-2
$$

are some complex numbers. Thus $O(1)$ could indicate different functions.

(I) $\mathcal{A}_{11}$ : it is a $(h d-2) \times(h d-2)$ matrix. Entries are coefficients of

$$
d \theta_{i}^{j}, d \epsilon_{m}, i \leq n-2,(i, j) \neq(0,1),(1,1), m=1, \cdots, 2 d
$$

for the vectors

$$
\pi_{1}^{*}\left(\psi_{i}\right), i=3, \cdots, h d .
$$

For this block matrix we use lemma 2.12 to obtain that its diagonal entries in the order of top-to-bottom are

$$
b_{3}, \cdots, b_{h d}
$$

where all $b_{i}=r_{0}^{2} b_{i}^{\prime}$ such that $b_{i}^{\prime}$ are polynomial functions on $U_{\widetilde{\mathbb{C}}^{(n+1)(d+1)}}$ that do not vanish at $\tilde{c}_{2}$. All the rest of entries are in the form

$$
r_{0}^{2} O(1)
$$

where $O(1)$ is defined in (2.81).

(II) $\mathcal{A}_{12}$ : it is $(h d-2) \times 1$ matrix. Entries are coefficients of $d r_{0}$ for the vectors

$$
\pi_{1}^{*}\left(\psi_{i}\right), i=3, \cdots, h d .
$$

So from top-to-bottom they are

$$
a_{3}, \cdots, a_{h d}
$$

where $a_{l}=r_{0} O(1)$.

(III) $\mathcal{A}_{13}$ : it is a $(h d-2) \times 6$ matrix. Entries are coefficients of

$$
d \theta_{0}^{1}, d \theta_{1}^{1}, d r_{1}, d r_{2}, d r_{n-1}, d r_{n}
$$

for the vectors

$$
\pi_{1}^{*}\left(\psi_{i}\right), i=3, \cdots, h d
$$

So they are all in the form

$$
r_{0}^{2} O(1)
$$

(IV) $\mathcal{A}_{21}$ : it is a $1 \times(h d-2)$ matrix. Entries are coefficients of

$$
d \theta_{i}^{j}, d \epsilon_{m}, i \leq n-2,(i, j) \neq(0,1),(1,1), m=1, \cdots, 2 d
$$


for the vector

$$
\pi_{1}^{*}\left(\psi_{h d+1}\right)
$$

So all entries are in the form

$$
r_{0}^{2} \mathrm{O}
$$

where $O$ is a polynomial function on $U_{\widetilde{\mathbb{C}}^{(n+1)(d+1)}}$.

(V) $\mathcal{A}_{22}$ : it is a $1 \times 1$ matrix. It is the coefficient of $d r_{0}$ for the vector,

$$
\pi_{1}^{*}\left(\psi_{h d+1}\right) .
$$

It is in the form

$$
r_{0} O_{h d+1}
$$

where $O_{h d+1}$ is a polynomial function on $U_{\widetilde{\mathbb{C}}(n+1)(d+1)}$ that does not vanish at $\tilde{c}_{2}$.

(VI) $\mathcal{A}_{23}$ : it is a $1 \times 6$ matrix. Entries are coefficients of

$$
d \theta_{0}^{1}, d \theta_{1}^{1}, d r_{1}, d r_{2}, d r_{n-1}, d r_{n}
$$

for the vector,

$$
\pi_{1}^{*}\left(\psi_{h d+1}\right)
$$

So all entries are in the form

$$
r_{0}^{2} \mathrm{O}
$$

where $O$ is a polynomial function on $U_{\widetilde{\mathbb{C}}^{(n+1)(d+1)}}$.

(VII) $\mathcal{A}_{31}$ : it is a $2 \times(h d-2)$ matrix. Entries are coefficients of

$$
d \theta_{i}^{j}, d \epsilon_{m}, i \leq n-2,(i, j) \neq(0,1),(1,1), m=1, \cdots, 2 d
$$

for the vectors

$$
\pi_{1}^{*}\left(d f_{2}\left(c\left(t_{1}\right)\right)\right), \pi_{1}^{*}\left(d f_{2}\left(c\left(t_{2}\right)\right)\right) .
$$

So the entries are all in the form

$$
r_{0} O
$$

where $O$ is a polynomial function on $U_{\tilde{\mathbb{C}}^{(n+1)(d+1)}}$.

(VIII) $\mathcal{A}_{32}$ : it is $2 \times 1$ matrix. Entries are the coefficients of $d r_{0}$ for the vectors

$$
\pi_{1}^{*}\left(d f_{2}\left(c\left(t_{1}\right)\right)\right), \pi_{1}^{*}\left(d f_{2}\left(c\left(t_{2}\right)\right)\right) .
$$

So they are in the form of

$$
O
$$

where $O$ is a polynomial function on $U_{\widetilde{\mathbb{C}}^{(n+1)(d+1)}}$.

(IVV) $\mathcal{A}_{33}$ : it is $2 \times 6$ matrix. Entries are the coefficients of

$$
d \theta_{1}^{1}, d \theta_{2}^{1}, d r_{1}, d r_{2}, d r_{n-1}, d r_{n}
$$


for the vectors

$$
\pi_{1}^{*}\left(d f_{2}\left(c\left(t_{1}\right)\right)\right), \pi_{1}^{*}\left(d f_{2}\left(c\left(t_{2}\right)\right)\right) .
$$

So all entries are in the forms of

$$
r_{0} O
$$

where $O$ is a polynomial function on $U_{\widetilde{\mathbb{C}}^{(n+1)(d+1)}}$.

(VV) $\mathcal{A}_{41}$ : it is a $2 \times(h d-2)$ matrix. Entries are coefficients of

$$
d \theta_{i}^{j}, d \epsilon_{m}, i \leq n-2,(i, j) \neq(0,1),(1,1), m=1, \cdots, 2 d
$$

for

$$
\pi_{1}^{*}\left(d f_{1}\left(c\left(t_{1}\right)\right)\right), \pi_{1}^{*}\left(d f_{1}\left(c\left(t_{2}\right)\right)\right) .
$$

All entries are in the form of

$$
r_{0} O
$$

where $O$ is a polynomial function on $U_{\widetilde{\mathbb{C}}^{(n+1)(d+1)}}$.

(VVI) $\mathcal{A}_{42}$ : it is a $2 \times 1$ matrix. Entries are the coefficients of $d r_{0}$ for the vector

$$
\pi_{1}^{*}\left(d f_{1}\left(c\left(t_{1}\right)\right)\right), \pi_{1}^{*}\left(d f_{1}\left(c\left(t_{2}\right)\right)\right) .
$$

(VVII) $\mathcal{A}_{43}$ : it is a $2 \times 6$ matrix. Entrices are the coefficients of

$$
d \theta_{0}^{1}, d \theta_{1}^{1}, d r_{1}, d r_{2}, d r_{n-1}, d r_{n}
$$

for the vector

$$
\pi_{1}^{*}\left(d f_{1}\left(c\left(t_{1}\right)\right)\right), \pi_{1}^{*}\left(d f_{1}\left(c\left(t_{2}\right)\right)\right) .
$$

All entries are in the form

$$
r_{0} O
$$

where $O$ is a polynomial function on $U_{\tilde{\mathbb{C}}^{(n+1)(d+1)}}$.

(VVIII) $\mathcal{A}_{51}$ : it is a $2 \times(h d-2)$ matrix. Entries are coefficients of

$$
d \theta_{i}^{j}, d \epsilon_{m}, i \leq n-2,(i, j) \neq(0,1),(1,1), m=1, \cdots, 2 d
$$

for

$$
\pi_{1}^{*}\left(d f_{0}\left(c\left(t_{1}\right)\right)\right), \pi_{1}^{*}\left(d f_{0}\left(c\left(t_{2}\right)\right)\right) .
$$

(VVIV) $\mathcal{A}_{52}$ : it is a $2 \times 1$ matrix. Entries are the coefficients of $d r_{0}$ for the vector

$$
\pi_{1}^{*}\left(d f_{0}\left(c\left(t_{1}\right)\right)\right), \pi_{1}^{*}\left(d f_{0}\left(c\left(t_{2}\right)\right)\right) .
$$

$(\mathrm{VVV}) \mathcal{A}_{53}$ : it is a $2 \times 6$ matrix. Entries are the coefficients of

$$
d \theta_{0}^{1}, d \theta_{1}^{1}, d r_{1}, d r_{2}, d r_{n-1}, d r_{n}
$$


for the vector

$$
\pi_{1}^{*}\left(d f_{0}\left(c\left(t_{1}\right)\right)\right), \pi_{1}^{*}\left(d f_{0}\left(c\left(t_{2}\right)\right)\right) .
$$

Next consider the function on $U_{\widetilde{\mathbb{C}}^{(n+1)(d+1)}}-E_{1}\left(\right.$ where $\left.r_{0} \neq 0\right)$,

$$
\mu(\tilde{c})=\frac{1}{\left(r_{0}\right)^{2 h d+1}}|\mathcal{A}|
$$

which is the determinant of the matrix

$$
\mathcal{A}_{r_{0}}=\left(\begin{array}{ccc}
\frac{1}{r_{0}^{2}} \mathcal{A}_{11} & \frac{1}{r_{0}^{2}} \mathcal{A}_{12} & \frac{1}{r_{0}^{2}} \mathcal{A}_{13} \\
\frac{1}{r_{0}} \mathcal{A}_{21} & \frac{1}{r_{0}} \mathcal{A}_{22} & \frac{1}{r_{0}} \mathcal{A}_{23} \\
\frac{1}{r_{0}} \mathcal{A}_{31} & \frac{1}{r_{0}} \mathcal{A}_{32} & \frac{1}{r_{0}} \mathcal{A}_{33} \\
\frac{1}{r_{0}} \mathcal{A}_{41} & \frac{1}{r_{0}} \mathcal{A}_{42} & \frac{1}{r_{0}} \mathcal{A}_{43} \\
\mathcal{A}_{51} & \mathcal{A}_{52} & \mathcal{A}_{53}
\end{array}\right) .
$$

To prove lemma 2.15 , it is sufficient to prove the determinant

$$
\left|\mathcal{A}_{r_{0}}\right| \text { at } r_{0}=0
$$

is non-zero. Unfortunately, the matrix $\mathcal{A}_{r_{0}}$ is not a well-defined at $\tilde{c}_{2}$ (i.e. $r_{0}=$ 0 ) because some entries involve $\frac{\left(t_{l}-\theta_{i}^{j}\right)}{r_{0}}$. But those terms do not show up in the computation of its determinant $\mu(\tilde{c})$. Hence $\mu(\tilde{c})$ can be continuously extended to $\tilde{c}_{2}$. Let's see this.

Computation: As before all expressions in the computation use coordinates in definition 2.11. Notice all entries in

$$
\left(\begin{array}{c}
\frac{1}{r_{0}^{2}} \mathcal{A}_{11} \\
\frac{1}{r_{0}} \mathcal{A}_{21} \\
\frac{1}{r_{0}} \mathcal{A}_{31} \\
\frac{1}{r_{0}} \mathcal{A}_{41} \\
\mathcal{A}_{51}
\end{array}\right)
$$

and

$$
\frac{1}{r_{0}^{2}} \mathcal{A}_{13}
$$

can be extended to the entire neighborhood $U_{\widetilde{\mathbb{C}}_{(n+1)(d+1)}}$, and when evaluated at $\tilde{c}_{2}$,

$$
\frac{1}{r_{0}^{2}} \mathcal{A}_{11}
$$

is a non-zero diagonal matrix (by lemma 2.12). Also notice that

$$
\left.\frac{1}{r_{0}^{2}} \mathcal{A}_{13}\right|_{\tilde{c}_{2}}=0 .
$$

Therefore we can perform the row operations on the matrix

$$
\mathcal{A}_{r_{0}}
$$


to reduce

$$
\left(\begin{array}{c}
\frac{1}{r_{0}} \mathcal{A}_{21} \\
\frac{1}{r_{0}} \mathcal{A}_{31} \\
\frac{1}{r_{0}} \mathcal{A}_{41} \\
\mathcal{A}_{51}
\end{array}\right)
$$

to zero matrix. Hence

$$
\mu(\tilde{c})=\frac{1}{\left(r_{0}\right)^{2 h d+1}}|\mathcal{A}|
$$

is a non-zero multiple of

$$
\left|\begin{array}{cc}
\frac{1}{r_{0}} \mathcal{A}_{22} & \frac{1}{r_{0}} \mathcal{A}_{23} \\
\frac{1}{r_{0}}\left(\mathcal{A}_{32}+O_{32}(1)\right) & \frac{1}{r_{0}} \mathcal{A}_{33} \\
\frac{1}{r_{0}}\left(\mathcal{A}_{42}+O_{42}(1)\right) & \frac{1}{r_{0}} \mathcal{A}_{43} \\
\mathcal{A}_{52}+\frac{1}{r_{0}} O_{52}(1) & \mathcal{A}_{53}
\end{array}\right|+O(1) .
$$

where $O(1), O_{i j}(1)$ represent the determinants of matrices, whose entries are local functions vanishing at $\tilde{c}_{2}$. Notice that in (2.91) the block matrices of first row and first column have sizes $1 \times 7$ and $7 \times 1$ respectively. Therefore by the linearity of determinants,

$$
\mu(\tilde{c})=\frac{1}{\rho}\left|\begin{array}{cc}
\frac{1}{r_{0}} \mathcal{A}_{22} & \frac{1}{r_{0}^{2}} \mathcal{A}_{23} \\
\mathcal{A}_{32} & \frac{1}{r_{0}} \mathcal{A}_{33} \\
\mathcal{A}_{42} & \frac{1}{r_{0}} \mathcal{A}_{43} \\
r_{0} \mathcal{A}_{52} & \mathcal{A}_{53}
\end{array}\right|+O(1)
$$

for some non-zero complex number $\rho$. Now all entries in (2.92) are well-defined functions on $U_{\widetilde{\mathbb{C}}^{(n+1)(d+1)}}$. Therefore it suffices to show the non-degeneracy of this $7 \times 7$ matrix

$$
\left.\left(\begin{array}{cc}
\frac{1}{r_{0}} \mathcal{A}_{22} & \frac{1}{r_{0}^{2}} \mathcal{A}_{23} \\
\mathcal{A}_{32} & \frac{1}{r_{0}} \mathcal{A}_{33} \\
\mathcal{A}_{42} & \frac{1}{r_{0}} \mathcal{A}_{43} \\
r_{0} \mathcal{A}_{52} & \mathcal{A}_{53}
\end{array}\right)\right|_{\tilde{c}_{2}}
$$

at the point $\tilde{c}_{2}$. Because $t_{h d+1}$ is generic, also $q$ is generic and $\tilde{\theta}_{i}^{j}$ are distinct, , it suffices to prove $6 \times 6$ matrix

$$
\left.\left(\begin{array}{c}
\frac{1}{r_{0}} \mathcal{A}_{33} \\
\frac{1}{r_{0}} \mathcal{A}_{43} \\
\mathcal{A}_{53}
\end{array}\right)\right|_{\tilde{c}_{2}}
$$

is non-degenerate.

Let $\lambda_{1}$ be the determinant of (2.94). Also let

$$
g_{i}(t)=\left.\left(\frac{1}{r_{0}} \pi_{1}^{*}\left(\frac{z_{i} \partial f_{1}}{\partial z_{i}}\right)\right)\right|_{\tilde{c}_{2}(t)} .
$$


Using the coordinates in definition 2.11, we compute that this determinant

$$
\lambda_{1}=\left.\operatorname{det}\left(\begin{array}{c}
\frac{1}{r_{0}} \mathcal{A}_{33} \\
\frac{1}{r_{0}} \mathcal{A}_{43} \\
\mathcal{A}_{53}
\end{array}\right)\right|_{\tilde{c}_{2}}
$$

is equal to

$$
\lambda_{2}\left|\begin{array}{cccccc}
\frac{1}{t_{1}-\tilde{\theta}_{0}^{1}} & \frac{1}{t_{1}-\tilde{\theta}_{1}^{1}} & 1 & 1 & 1 & 1 \\
\frac{1}{t_{2}-\tilde{\theta}_{0}^{1}} & \frac{1}{t_{2}-\tilde{\theta}_{1}^{1}} & 1 & 1 & 1 & 1 \\
\frac{\partial \pi_{1}^{*}\left(f_{1}\right)\left(\tilde{c}_{2}\left(t_{1}\right)\right)}{r_{0} \theta_{0}^{1}} & \frac{\partial \pi_{1}^{*}\left(f_{1}\right)\left(\tilde{c}_{2}\left(t_{1}\right)\right)}{r_{0} \partial \theta_{1}^{1}} & g_{1}\left(t_{1}\right) & g_{2}\left(t_{1}\right) & g_{n-1}\left(t_{1}\right) & g_{n}\left(t_{1}\right) \\
\frac{\partial \pi_{1}^{*}\left(f_{1}\right)\left(\tilde{c}_{2}\left(t_{2}\right)\right)}{r_{0} \partial \partial \theta_{1}^{1}} & \frac{\partial \pi_{1}^{*}\left(f_{1}\right)\left(\tilde{c}_{2}\left(t_{2}\right)\right)}{r_{0} \partial \theta_{1}^{1}} & g_{1}\left(t_{2}\right) & g_{2}\left(t_{2}\right) & g_{n-1}\left(t_{2}\right) & g_{n}\left(t_{2}\right) \\
\frac{\partial \pi_{1}^{*}\left(f_{0}\right)\left(\tilde{c}_{2}\left(t_{1}\right)\right)}{\partial \theta_{1}^{1}} & \frac{\partial \pi_{1}^{*}\left(f_{0}\right)\left(\tilde{c}_{2}\left(t_{1}\right)\right)}{\partial \theta_{1}^{1}} & \left.\left(z_{1} \frac{\partial f_{0}}{\partial z_{1}}\right)\right|_{\tilde{c}_{2}\left(t_{1}\right)} & \left.\left(z_{2} \frac{\partial f_{0}}{\partial z_{2}}\right)\right|_{\tilde{c}_{2}\left(t_{1}\right)} & \left.\left(z_{n-1} \frac{\partial f_{0}}{\partial z_{n-1}}\right)\right|_{\tilde{c}_{2}\left(t_{1}\right)} & \left.\left(z_{n} \frac{\partial f_{0}}{\partial z_{n}}\right)\right|_{\tilde{c}_{2}\left(t_{1}\right)} \\
\frac{\partial \pi_{1}^{*}\left(f_{0}\right)\left(\tilde{c}_{2}\left(t_{2}\right)\right)}{\partial \theta_{0}^{1}} & \frac{\partial \pi_{1}^{*}\left(f_{0}\right)\left(\tilde{c}_{2}\left(t_{2}\right)\right)}{\partial \theta_{1}^{1}} & \left.\left(z_{1} \frac{\partial f_{0}}{\partial z_{1}}\right)\right|_{\tilde{c}_{2}\left(t_{2}\right)} & \left.\left(z_{2} \frac{\partial f_{0}}{\partial z_{2}}\right)\right|_{\tilde{c}_{2}\left(t_{2}\right)} & \left.\left(z_{n-1} \frac{\partial \partial f_{0}}{\partial z_{n-1}}\right)\right|_{\tilde{c}_{2}\left(t_{2}\right)} & \left.\left(z_{n} \frac{\partial f_{0}}{\partial z_{n}}\right)\right|_{\tilde{c}_{2}\left(t_{2}\right)}
\end{array}\right|
$$

where

$$
\lambda_{2}=\left.\prod_{i=1}^{2}\left(\frac{\pi_{1}^{*}\left(f_{2}\right)}{y_{1} y_{2} y_{n-1} y_{n} r_{0}}\right)\right|_{\tilde{c}_{2}\left(t_{i}\right)} \neq 0 .
$$

Because $q$ is generic, the two middle row vectors of (2.96)

$$
\begin{array}{llllll}
\left(\frac{\partial \pi_{1}^{*}\left(f_{1}\right)\left(\tilde{c}_{2}\left(t_{1}\right)\right)}{r_{0} \partial \theta_{0}^{1}}\right. & \frac{\partial \pi_{1}^{*}\left(f_{1}\right)\left(\tilde{c}_{2}\left(t_{1}\right)\right)}{r_{0} \partial \theta_{1}^{1}} & g_{1}\left(t_{1}\right) & g_{2}\left(t_{1}\right) & g_{n-1}\left(t_{1}\right) & \left.g_{n}\left(t_{1}\right)\right) \\
\left(\frac{\partial \pi_{1}^{*}\left(f_{1}\right)\left(\tilde{c}_{2}\left(t_{2}\right)\right)}{r_{0} \partial \theta_{0}^{1}}\right. & \frac{\partial \pi_{1}^{*}\left(f_{1}\right)\left(\tilde{c}_{2}\left(t_{2}\right)\right)}{r_{0} \partial \theta_{1}^{1}} & g_{1}\left(t_{2}\right) & g_{2}\left(t_{2}\right) & g_{n-1}\left(t_{2}\right) & \left.g_{n}\left(t_{2}\right)\right)
\end{array}
$$

evaluated at $\tilde{c}_{2}$ are generic vectors in $\mathbb{C}^{6}$. Thus to show (2.96) is non-zero, it suffices to show that the minor

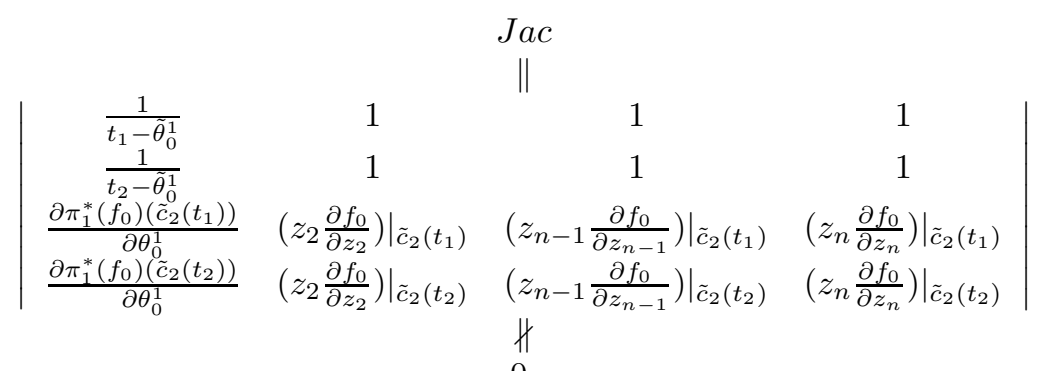

0 .

(Remove the 2nd and 3rd columns in (2.96)). We further compute to have

$$
\begin{gathered}
J a c \\
\left(\frac{1}{t_{1}-\hat{\theta}_{0}^{1}}-\frac{1}{t_{2}-\hat{\theta}_{0}^{1}}\right)\left|\begin{array}{ccc}
1 & \| & 1 \\
\left.\left(z_{2} \frac{\left.\partial f_{0}\right)}{\partial z_{2}}\right)\right|_{c_{2}\left(t_{1}\right)} & \left.\left(z_{n-1} \frac{\left.\partial f_{0}\right)}{\partial z_{n}-1}\right)\right|_{c_{2}\left(t_{1}\right)} & \left.\left(z_{n} \frac{\left.\partial f_{0}\right)}{\partial z_{n}}\right)\right|_{c_{2}\left(t_{1}\right)} \\
\left.\left(z_{2} \frac{\left.\partial f_{0}\right)}{\partial z_{2}}\right)\right|_{c_{2}\left(t_{2}\right)} & \left.\left(z_{n-1} \frac{\left.\partial f_{0}\right)}{\partial z_{n-1}}\right)\right|_{c_{2}\left(t_{2}\right)} & \left.\left(z_{n} \frac{\left.\partial f_{0}\right)}{\partial z_{n}}\right)\right|_{c_{2}\left(t_{2}\right)}
\end{array}\right| .
\end{gathered}
$$


Suppose $J a c=0$. Because $q$ is generic, $\left(t_{1}, t_{2}\right)$ must a generic point in $\mathbb{C}^{2}$. Hence

$$
c_{2}(t)=\left\{\left[0, c_{2}^{1}(t), \cdots, c_{2}^{n-1}(t), c_{2}^{n}(t)\right]\right\}
$$

lies in the hypersurface

$$
z_{2} \frac{\partial f_{0}}{\partial z_{2}}+z_{n-1} \frac{\partial f_{0}}{\partial z_{n-1}}+z_{n} \frac{\partial f_{0}}{\partial z_{n}}=0 .
$$

Similarly we can conclude that $\left\{c_{2}(t)\right\}=C_{2}$ (image of $\mathbf{P}^{1}$ ) lies in the hypersurface

$$
H_{j}=\left\{\Sigma_{i \neq j} z_{i} \frac{\partial f_{0}}{\partial z_{i}}=0\right\}
$$

for $j=1, \cdots, n$. Since $f_{0}$ is generic,

$$
\cap_{j} H_{j}
$$

is finite. Then $C_{2}$ has to be a point. This contradicts our assumption for the step 1 .

Hence

$$
\left|\begin{array}{ccc}
1 & 1 & 1 \\
\left.\left(z_{2} \frac{\left.\partial f_{0}\right)}{\partial z_{2}}\right)\right|_{c_{2}\left(t_{1}\right)} & \left.\left(z_{n-1} \frac{\left.\partial f_{0}\right)}{\partial z_{n}-1}\right)\right|_{c_{2}\left(t_{1}\right)} & \left.\left(z_{n} \frac{\left.\partial f_{0}\right)}{\partial z_{n}}\right)\right|_{c_{2}\left(t_{1}\right)} \\
\left.\left(z_{2} \frac{\left.\partial f_{0}\right)}{\partial z_{2}}\right)\right|_{c_{2}\left(t_{2}\right)} & \left.\left(z_{n-1} \frac{\left.\partial f_{0}\right)}{\partial z_{n-1}}\right)\right|_{c_{2}\left(t_{2}\right)} & \left.\left(z_{4} \frac{\left.\partial f_{0}\right)}{\partial z_{4}}\right)\right|_{c_{2}\left(t_{2}\right)}
\end{array}\right| \neq 0
$$

Therefore

$$
J a c \neq 0 .
$$

This completes the proof of lemma 2.15, thus the first step of lemma 2.14.

Step 2: The following formulation of step 2 can be viewed as the generalization of step 1. Both steps have the same formulation after the blow-ups.

Let's assume $\tilde{\theta}_{i}^{j}$ are not distinct. Notice $\omega$ depends on the choice of $t_{i}, i=$ $1, \cdots, h d+1$. The main idea we would like to get across in this paper is that for generic choice of $t_{i}, \omega$ is non-zero near $c_{2}$ (excluding $c_{2}$ ). However it seems to be completely helpless in the computation of $\omega$ for generic $t_{i}$. This situation changes if we use a special choice of $t_{i}$ such as in step 1 , in which case the Jacobian matrix in $\omega$ breaks down to manageable block matrices in (2.80). The choice of such $t_{i}$ requires that $\tilde{\theta}_{i}^{j}$ are distinct. If they aren't (i.e. $\tilde{c}_{2}$ has multiple intersection points with coordinates' plane), the step 1 will fail because the main block in (2.80) becomes degenerate. So to resolve this, we will use successive blow-ups in the following to reduce the multiple $\tilde{\theta}_{i}^{j}$ to distinct $\tilde{\theta}_{i}^{j}$. The pull-back of $\omega$ by the blow-ups for generic $t_{i}$ is exactly the same as (5.10) which is that in step 1.

Let's discuss the blow-ups. They are all analytic which means the total spaces and the centers are analytic in an analytic neighborhood. Suppose we already have the blow-up $\tilde{\mathbb{C}}^{h(d+1)}$ as in (2.62). Recall $\tilde{c}_{2}^{i} \in H^{0}\left(\mathcal{O}_{\mathbf{P}^{1}}(d)\right)$ are sections such that

$$
\operatorname{div}\left(\tilde{c}_{2}^{i}\right)=\sum_{j=1}^{d} \tilde{\theta}_{i}^{j}
$$


for $i=0, \cdots, n$. We may assume multiple zeros are

$$
\tilde{\theta}_{\alpha}^{\beta}
$$

among all $\tilde{\theta}_{i}^{j}$, where $\alpha, \beta$ are finite numbers less than $h$ and $d+1$ respectively. Let

$$
m\left(\tilde{c}_{2}\right)
$$

be the number of the pairs $(\alpha, \beta)$ which is the number of multiple zeros $\theta_{i}^{j}$. The worst case is $m\left(\tilde{c}_{2}\right)=h d$, in which case $\tilde{c}_{2}$ represents a constant map $\mathbf{P}^{1} \rightarrow \mathbf{P}^{n}$. This multiplicity is well-defined for the map $\tilde{c}_{2}$, but depends on the coordinates $z_{i}$. Next we define the successive blow-ups which are controlled by the multiplicity $m\left(\tilde{c}_{2}\right)$.

First blow-up. First we should note the coordinates $r_{i}, \theta_{i}^{j}$ are well-defined analytic coordinates for $\tilde{M}$ around the point $\tilde{c}_{2}$, even if $\theta_{i}^{j}$ are not distinct 7 We define $B_{2}$ to be the analytic subvariety

$$
\left\{\tilde{\theta}_{\alpha}^{\beta}-\theta_{\alpha}^{\beta}=0, \text { for all } \alpha, \beta\right\} .
$$

of an analytic open set $U_{\widetilde{\mathbb{C}} h(d+1)}$ of $\tilde{\mathbb{C}}^{h(d+1)}$, centered around $\tilde{c}_{2}$. For the simplicity we denote $U_{\widetilde{\mathbb{C}}^{h(d+1)}}$ by $\tilde{\mathbb{C}}^{h(d+1)}$. We blow-up $\tilde{\mathbb{C}}^{h(d+1)}$ along $B_{2}$ to obtain the first blow-up map

$$
N \stackrel{\pi_{2}}{\rightarrow} \tilde{\mathbb{C}}^{h(d+1)} .
$$

Let $\tilde{c}_{2}^{(1)}$ be the inverse of $\tilde{c}_{2}$ in the strict transform of $\tilde{P}\left(\Gamma_{\mathbb{L}_{1}}\right)$. Using the coordinates $\theta_{i}^{j}, r_{i}$ above, we can find an open set $U_{N}$ centered around $\tilde{c}_{2}^{(1)}$ such that $U_{N}$ is analytically isomorphic to

$$
U_{N} \simeq \mathbb{C}^{d+1} \times \cdots \times \mathbb{C}^{d+1},
$$

where each copy $\mathbb{C}^{d+1}$ corresponds to $\theta_{i}^{j}$ with the same $i$. Precisely the i-th copy $\mathbb{C}^{d+1}$ in (2.102) is determined by the defining equations

$$
\pi_{2}^{*}\left(\theta_{i}^{j}\right)=0, \pi_{2}^{*}\left(r_{i}\right)=0 .
$$

Next fix the isomorphism,

$$
\begin{array}{ccc}
\mathbb{C}^{d+1} & \simeq & H^{0}\left(\mathcal{O}_{\mathbf{P}^{1}}(d)\right) \\
\left(r_{i},\left(\theta_{i}^{0}\right)^{(1)}, \cdots,\left(\theta_{i}^{d}\right)^{(1)}\right) & \rightarrow & r_{i} \sum_{j=0}^{d}\left(t-\left(\theta_{i}^{j}\right)^{(1)}\right) .
\end{array}
$$

Then we obtain the new evaluation $\operatorname{map} Z^{(1)}$,

$$
\begin{array}{ccc}
U_{N} \times \mathbf{P}^{1} & \stackrel{Z^{(1)}}{\rightarrow} & \mathbf{P}^{n} \\
\left(\tilde{c}^{(1)}, t\right) & \rightarrow & {\left[\tilde{c}^{(1)}(t)\right] .}
\end{array}
$$

The map $Z^{(1)}$ turns $U_{N}$ into a family of maps 8 ,

$$
\mathbf{P}^{1} \rightarrow \mathbf{P}^{n}
$$

\footnotetext{
7 This is actually true in a Zariski topology. The analytic blow-ups we are using here can be replaced by the algebraic ones.

${ }^{8}$ Rational curves in this family do not lie on hypersurfaces in the family $\mathbb{L}$. So they are not from the family $\tilde{P}\left(\Gamma_{\mathbb{L}}\right)$. But their transformations outside of the exceptional divisor of the blow-up do. This transformation is nothing else but the isomorphism of the blow-up outside of the exceptional divisor through the identification (2.103). Then $\tilde{c}_{2}^{(1)}$ is a specialization of the generic member in this family $U_{N}$ of rational curves.
} 
Using (2.102) and (2.103), we let

$$
\left(\tilde{c}_{2}\right)^{(1)}=\left(\left(\tilde{c}_{2}\right)_{0}^{(1)}(t), \cdots,\left(\tilde{c}_{2}\right)_{n}^{(1)}(t)\right),
$$

Continuing blow-ups. If $\tilde{c}_{2}^{(1)}(t)$ still has multiple zeros with the planes $\left\{z_{i}=0\right\}$, i.e. $\left(\tilde{c}_{2}\right)_{i}^{(1)}(t)=0$ for all $i$ have multiple zeros or equivalently

$$
m\left(\tilde{c}_{2}^{(1)}\right)>1
$$

we continue the blow-ups along the multiple zeros as above. This type of blow-ups can continue. The last blow-up is obtained when the multiplicity $m\left(\tilde{c}_{2}^{(\kappa)}\right)$ is reduced to 1 ( need to repeat (2.102) and (2.103) in each blow-up to have the well-defined $\left.m\left(\tilde{c}_{2}^{(\kappa)}\right)\right)$. To see that $m\left(\tilde{c}_{2}\right)$ will be reduced to 1 (i.e. the blow-ups will stop), we go back to the first blow-up. Let $\left(P\left(\Gamma_{\mathbb{L}_{1}}\right)\right)^{(1)}$ be the strict transform of $\tilde{P}\left(\Gamma_{\mathbb{L}_{1}}\right)$ under the first blow-up, and $\tilde{c}_{2}^{(1)}$ be a chosen inverse of $\tilde{c}_{2}$ in $\left(P\left(\Gamma_{\mathbb{L}_{1}}\right)\right)^{(1)}$. Since $\tilde{P}\left(\Gamma_{\mathbb{L}_{1}}\right)$ does not lie in the $B_{2}$, the exceptional divisor $\mathcal{D}$ of $\left(P\left(\Gamma_{\mathbb{L}_{1}}\right)\right)^{(1)}$ does not lie in the tangent bundle of $B_{2}$ ( $\mathcal{D}$ is a subvariety in the projectivization of the normal bundle of $B_{2}$ ). Therefore $\tilde{c}_{2}^{(1)}$ when regarded as a map $\mathbf{P}^{1} \rightarrow \mathbf{P}^{4}$ has multiplicity

$$
m\left(\tilde{c}_{2}^{(1)}\right)
$$

strictly less than $m\left(\tilde{c}_{2}\right)$. After such successive blow-ups, we obtain the inverse $\tilde{c}_{2}^{(\kappa)}$ of $\tilde{c}_{2}$ whose multiplicity

$$
m\left(\tilde{c}_{2}^{(\kappa)}\right)
$$

is 1 . We let $\pi_{3}$ be the composition of all such blow-ups. So we obtain the birational map

$$
\left(\tilde{\mathbb{C}}^{h(d+1)}\right)^{(\kappa)} \stackrel{\pi_{3}}{\rightarrow} \tilde{\mathbb{C}}^{h(d+1)}
$$

As above, there are a determined open set $U^{(\kappa)}$ of $\left(\tilde{\mathbb{C}}^{h(d+1)}\right)^{(\kappa)}$, centered at $\tilde{c}_{2}^{(\kappa)}$, and a determined analytic isomorphism

$$
U^{(\kappa)} \simeq\left(\mathbb{C}^{d+1}\right)^{\oplus h}
$$

We denote the natural affine coordinates of

$$
\left(\mathbb{C}^{d+1}\right)^{\oplus 5}
$$

in $(2.105)$ by $c^{(\kappa)}$.

Computation of the pull-back by blow-ups. Next we apply the successive blow-ups $\pi_{3}$ to prove the lemma. We would like to show that after the blow-ups we have the same set-up as for the step 1 . Let $\tilde{P}\left(\Gamma_{\mathbb{L}}\right)^{(\kappa)}$ be the strict transform of $\tilde{P}\left(\Gamma_{\mathbb{L}}\right)$ under $\pi_{3}$. Then $U^{(\kappa)} \cap \tilde{P}\left(\Gamma_{\mathbb{L}}\right)^{(\kappa)}$ parametrizes a family of rational curves in $\mathbf{P}^{4}$ (these rational curves do not lie on the hypersurfaces in $\mathbb{L}$ ).

These successive blow-ups can be stated in the following diagram 


$$
\begin{array}{ccccccc}
\tilde{P}\left(\Gamma_{\mathbb{L}}\right)^{(\kappa)} & \stackrel{P}{\leftarrow} & W & \subset & \left(\tilde{\mathbb{C}}^{h(d+1)}\right)^{(\kappa)} \times \mathbb{L} & \stackrel{\text { Proj. }}{\rightarrow} & \mathbb{L} \\
\downarrow \pi_{3} & & \downarrow & & \downarrow \pi_{3} \times \text { identity } & & \\
\tilde{P}\left(\Gamma_{\mathbb{L}}\right) & \stackrel{P}{\leftarrow} & \Gamma_{\mathbb{L}} & \subset & \tilde{\mathbb{C}}^{h(d+1)} \times \mathbb{L} & \stackrel{\text { Proj. }}{\rightarrow} & \mathbb{L}
\end{array}
$$

where $W$ is the strict transform of $\Gamma_{\mathbb{L}}$ and $\pi_{3}$ is birational.

Restrict this diagram to a small neighborhood $U^{(\kappa)}$ of $\tilde{c}_{2}^{(\kappa)}$, we have the map $\pi_{3}$

$$
\begin{array}{llc}
U^{(\kappa)} & \rightarrow & \tilde{\mathbb{C}}^{h(d+1)} \\
\tilde{c}^{(\kappa)} & \rightarrow & \tilde{c} .
\end{array}
$$

Notice the map $\tilde{c}^{(\kappa)} \rightarrow \tilde{c}$ is the restriction of $\pi_{3}$ which is an isomorphism outside of $\left(\pi_{1} \circ \pi_{3}\right)^{-1}\left(B_{2}\right)$. This diagram does not explain when $\pi_{3}$ stops. The termination of the successive blow-ups depends on the higher orders of $\tilde{P}\left(\Gamma_{\mathbb{L}}\right)^{(\kappa)}$ explained above. In addition blow-ups themselves also rely on the natural coordinates of $M$. Next we explain the choice of our new $t_{i}$ which are different from those in step 1 because of the multiplicity. Let

$$
f_{3}=z_{0} \cdots z_{n-2}\left(\delta_{1} q+\delta_{2} z_{n-1} z_{n}\right)
$$

and $t_{1}, t_{2}$ be generic numbers satisfying

$$
\left|\begin{array}{ll}
\left.q\right|_{\tilde{c}_{2}^{(\kappa)}\left(t_{1}\right)} & \left(\tilde{c}_{2}^{n-1}\right)^{(\kappa)}\left(t_{1}\right)\left(\tilde{c}_{2}^{n}\right)^{(\kappa)}\left(t_{1}\right) \\
\left.q\right|_{\tilde{c}_{2}^{(\kappa)}\left(t_{2}\right)} & \left.\left(\tilde{c}_{2}^{n-1}\right)^{(\kappa)}\left(t_{2}\right)\left(\tilde{c}_{2}^{n}\right)^{(\kappa)}\left(t_{2}\right)\right)
\end{array}\right|=0 .
$$

where $\tilde{c}_{2}^{(\kappa)}=\left(\left(\tilde{c}_{2}^{0}\right)^{(\kappa)}, \cdots,\left(\tilde{c}_{2}^{n}\right)^{(\kappa)}\right)$, and

$$
\delta_{1}=\left|\begin{array}{ll}
f_{0}\left(\tilde{c}_{2}^{(\kappa)}\left(t_{1}\right)\right) & f_{2}\left(\tilde{c}_{2}^{(\kappa)}\left(t_{1}\right)\right) \\
f_{0}\left(\tilde{c}_{2}^{(\kappa)}\left(t_{2}\right)\right) & f_{2}\left(\tilde{c}_{2}^{(\kappa)}\left(t_{2}\right)\right)
\end{array}\right|, \quad \delta_{2}=\left|\begin{array}{cc}
f_{1}\left(\tilde{c}_{2}^{(\kappa)}\left(t_{1}\right)\right) & f_{0}\left(\tilde{c}_{2}^{(\kappa)}\left(t_{1}\right)\right) \\
f_{1}\left(\tilde{c}_{2}^{(\kappa)}\left(t_{2}\right)\right) & \left.f_{0}\left(\tilde{c}_{2}^{(\kappa)}\right)\left(t_{2}\right)\right)
\end{array}\right| .
$$

Let $\left(\tilde{\theta}_{i}^{j}\right)^{(\kappa)}$ be the zeros of $\left(\tilde{c}_{2}^{i}\right)^{(\kappa)}(t)=0, i=0, \cdots, n$. Then let $t_{3}, \cdots, t_{h d}$ be zeros of

$$
\begin{gathered}
\left.f_{3}(z)\right|_{\tilde{c}_{2}^{(\kappa)}(t)} \\
\left.\| \tilde{c}_{2}^{0}\right)^{(\kappa)}(t) \cdots\left(\tilde{c}_{2}^{n-2}\right)^{(\kappa)}(t)\left(\left.\delta_{1} q\right|_{\tilde{c}_{2}^{(\kappa)}(t)}+\delta_{2}\left(\tilde{c}_{2}^{n-1}\right)^{(\kappa)}(t)\left(\tilde{c}_{2}^{n}\right)^{(\kappa)}(t)\right) \\
\|
\end{gathered}
$$

other than $\left(\tilde{\theta}_{0}^{1}\right)^{(\kappa)},\left(\tilde{\theta}_{1}^{1}\right)^{(\kappa)}$. Let $t_{h d+1}$ be generic.

With such a special choice of $t_{i}$, we can repeat the step 1 for the same quintics $f_{i}(z)$ to construct $\omega^{(\kappa)}$ where $\omega^{(\kappa)}$ has the current choice of $t_{i}$ and the variables are $\tilde{c}^{(\kappa)}$ for $U^{(\kappa)}$. Specifically, we define

$$
\phi_{i}^{(\kappa)}=d\left|\begin{array}{lll}
f_{2}\left(\tilde{c}^{(\kappa)}\left(t_{i}\right)\right) & f_{1}\left(\tilde{c}^{(\kappa)}\left(t_{i}\right)\right) & f_{0}\left(\tilde{c}^{(\kappa)}\left(t_{i}\right)\right) \\
f_{2}\left(\tilde{c}^{(\kappa)}\left(t_{1}\right)\right) & f_{1}\left(\tilde{c}^{(\kappa)}\left(t_{1}\right)\right) & f_{0}\left(\tilde{c}^{(\kappa)}\left(t_{1}\right)\right) \\
f_{2}\left(\tilde{c}^{(\kappa)}\left(t_{2}\right)\right) & f_{1}\left(\tilde{c}^{(\kappa)}\left(t_{2}\right)\right) & f_{0}\left(\tilde{c}^{(\kappa)}\left(t_{2}\right)\right)
\end{array}\right|
$$

for $i=3, \cdots, h d+1$

$$
\omega^{(\kappa)}=\wedge_{i=3}^{h d+1} \phi_{i}^{(\kappa)} \in H^{0}\left(\Omega_{U^{(\kappa)}}\right) .
$$


Then we repeat the same process (but without blow-up $\pi_{1}$ ) in step 1 to construct the Jacobian matrix

$$
\mathcal{A}^{(\kappa)}
$$

with respect to $\omega^{(\kappa)}$ and the point $\tilde{c}_{2}^{(\kappa)}$. Then we use the same process in step 1 to calculate $\operatorname{det}\left(\mathcal{A}^{(\kappa)}\right)$. We obtain same result as in lemma 5.4, i.e.

$$
\operatorname{det}\left(\mathcal{A}^{(\kappa)}\right) \neq 0 .
$$

at the origin $\tilde{c}_{2}^{(\kappa)}$. Next we discuss the relation between $\operatorname{det}(\mathcal{A})$ and $\operatorname{det}\left(\mathcal{A}^{(\kappa)}\right)$. We would like see that, as in step $1 \operatorname{det}(\mathcal{A})$ is for the Jacobian of the generic points of $\tilde{P}\left(\Gamma_{L}\right), \operatorname{det}\left(\mathcal{A}^{(\kappa)}\right)$ now is for the Jacobian of the generic points of $\left(\tilde{P}\left(\Gamma_{L}\right)\right)^{(\kappa)}$. Notice that $\pi_{3}$ is an isomorphism outside of exceptional divisor $\pi_{3}^{-1}\left(B_{2}\right)$. So the equations

$$
\left|\begin{array}{lll}
f_{2}\left(\tilde{c}^{(\kappa)}\left(t_{i}\right)\right) & f_{1}\left(\tilde{c}^{(\kappa)}\left(t_{i}\right)\right) & f_{0}\left(\tilde{c}^{(\kappa)}\left(t_{i}\right)\right) \\
f_{2}\left(\tilde{c}^{(\kappa)}\left(t_{1}\right)\right) & f_{1}\left(\tilde{c}^{(\kappa)}\left(t_{1}\right)\right) & f_{0}\left(\tilde{c}^{(\kappa)}\left(t_{1}\right)\right) \\
f_{2}\left(\tilde{c}^{(\kappa)}\left(t_{2}\right)\right) & f_{1}\left(\tilde{c}^{(\kappa)}\left(t_{2}\right)\right) & f_{0}\left(\tilde{c}^{(\kappa)}\left(t_{2}\right)\right)
\end{array}\right|=0
$$

$i=3, \cdots, h d+1$ defines the scheme

$$
\tilde{P}\left(\Gamma_{\mathbb{L}}\right)^{(\kappa)}=\pi_{3}^{-1}\left(\tilde{P}\left(\Gamma_{\mathbb{L}}\right)\right)
$$

in $U^{(\kappa)}-\pi_{3}^{-1}\left(B_{2}\right)$. This shows that on the open set

$$
U^{(\kappa)} \cap \tilde{P}\left(\Gamma_{\mathbb{L}}\right)^{(\kappa)}-\pi_{3}^{-1}\left(B_{2}\right)
$$

where $g$ is a function nowhere zero on

$$
\tilde{P}\left(\Gamma_{\mathbb{L}}\right)^{(\kappa)}-\pi_{3}^{-1}\left(B_{2}\right) .
$$

Because of $(2.111), \operatorname{det}(\mathcal{A}) \neq 0$ at a point in

$$
\pi_{1} \circ \pi_{3}\left(U^{(\kappa)}\right) \cap P\left(\Gamma_{\mathbb{L}}\right)-\left\{c_{2}\right\} .
$$

This proves lemma 2.14 .

Proof. of lemma 2.13: We first calculate 1-form $\phi_{i}$ on $M$ evaluated at general $c_{g} \in M$. For $i=3, \cdots, h d+1$,

$$
\begin{aligned}
& \phi_{i}=d\left|\begin{array}{lll}
f_{2}\left(c\left(t_{i}\right)\right) & f_{1}\left(c\left(t_{i}\right)\right) & f_{0}\left(c\left(t_{i}\right)\right) \\
f_{2}\left(c\left(t_{1}\right)\right) & f_{1}\left(c\left(t_{1}\right)\right) & f_{0}\left(c\left(t_{1}\right)\right) \\
f_{2}\left(c\left(t_{2}\right)\right) & f_{1}\left(c\left(t_{2}\right)\right) & f_{0}\left(c\left(t_{2}\right)\right)
\end{array}\right| \\
& \begin{array}{ll}
f_{0}\left(c_{g}\left(t_{1}\right)\right) & f_{2}\left(c_{g}\left(t_{1}\right)\right) \\
f_{0}\left(c_{g}\left(t_{2}\right)\right) & f_{2}\left(c_{g}\left(t_{2}\right)\right)
\end{array}\left|d f_{1}\left(c\left(t_{i}\right)\right)+\right| \begin{array}{ll}
f_{2}\left(c_{g}\left(t_{1}\right)\right) & f_{1}\left(c_{g}\left(t_{1}\right)\right) \\
f_{2}\left(c_{g}\left(t_{2}\right)\right) & f_{1}\left(c_{g}\left(t_{2}\right)\right)
\end{array} \mid d f_{0}\left(c\left(t_{i}\right)\right) \\
& +\left|\begin{array}{ll}
f_{1}\left(c_{g}\left(t_{1}\right)\right) & f_{0}\left(c_{g}\left(t_{1}\right)\right) \\
f_{1}\left(c_{g}\left(t_{2}\right)\right) & f_{0}\left(c_{g}\left(t_{2}\right)\right)
\end{array}\right| d f_{2}\left(c\left(t_{i}\right)\right)+\sum_{l=0, j=1}^{l=2, j=2} h_{l j}^{i}\left(c_{g}\right) d f_{l}\left(c\left(t_{j}\right)\right)
\end{aligned}
$$


where $h_{l j}^{i}$ are polynomials in $c$.

The lemma 2.14 implies that for a generic choice of

$$
f_{0}, f_{1}, f_{2}, c_{g}, t_{1}, \cdots, t_{h d+1},
$$

$$
\begin{aligned}
& \begin{array}{ll}
f_{0}\left(c_{g}\left(t_{1}\right)\right) & f_{2}\left(c_{g}\left(t_{1}\right)\right) \\
f_{0}\left(c_{g}\left(t_{2}\right)\right) & f_{2}\left(c_{g}\left(t_{2}\right)\right)
\end{array}\left|d f_{1}\left(c\left(t_{i}\right)\right)+\right| \begin{array}{ll}
f_{2}\left(c_{g}\left(t_{1}\right)\right) & f_{1}\left(c_{g}\left(t_{1}\right)\right) \\
f_{2}\left(c_{g}\left(t_{2}\right)\right) & f_{1}\left(c_{g}\left(t_{2}\right)\right)
\end{array} \mid d f_{0}\left(c\left(t_{i}\right)\right) \\
& +\left|\begin{array}{ll}
f_{1}\left(c_{g}\left(t_{1}\right)\right) & f_{0}\left(c_{g}\left(t_{1}\right)\right) \\
f_{1}\left(c_{g}\left(t_{2}\right)\right) & f_{0}\left(c_{g}\left(t_{2}\right)\right)
\end{array}\right| d f_{2}\left(c\left(t_{i}\right)\right)
\end{aligned}
$$

for $i=3, \cdots, h d+1$, and

$$
d f_{l}\left(c\left(t_{j}\right)\right), l=0,1,2, j=1,2
$$

are $h d+5$ linearly independent vectors in $\left(T_{c_{g}} M\right)^{*}$ for a generic $c_{g} \in P\left(\Gamma_{\mathbb{L}}\right)$ (not at special point $\left.c_{2}\right)$. i.e. they form a basis of the vector space $\left(T_{c_{g}} M\right)^{*}$.

This implies the set of 1 -forms $\left\{\phi_{i}\right\}_{i=3, \cdots, h d+1}$ is a linearly independent set in $\left(T_{c_{g}} M\right)^{*}$ for generic $c_{g} \in\left(T_{c_{g}} M\right)^{*}$. Thus $\omega$ is nowhere zero when it is evaluated at generic points of $P\left(\Gamma_{\mathbb{L}}\right)$. The lemma 2.13 is proved.

$\mathrm{C}$

\subsubsection{Ranks of differential sheaves}

Proof. of proposition 1.3: Let $\mathcal{N}$ be the submodule of global sections, $H^{0}\left(\Omega_{M}\right)$ generated by elements

$$
\phi_{i}=d\left|\begin{array}{lll}
f_{2}\left(c\left(t_{1}\right)\right) & f_{1}\left(c\left(t_{1}\right)\right) & f_{0}\left(c\left(t_{1}\right)\right) \\
f_{2}\left(c\left(t_{2}\right)\right) & f_{1}\left(c\left(t_{2}\right)\right) & f_{0}\left(c\left(t_{2}\right)\right) \\
f_{2}\left(c\left(t_{i}\right)\right) & f_{1}\left(c\left(t_{i}\right)\right) & f_{0}\left(c\left(t_{i}\right)\right)
\end{array}\right|
$$

for $i=3, \cdots, h d+1$. Recall that

$$
\left|\begin{array}{lll}
f_{2}\left(c\left(t_{1}\right)\right) & f_{1}\left(c\left(t_{1}\right)\right) & f_{0}\left(c\left(t_{1}\right)\right) \\
f_{2}\left(c\left(t_{2}\right)\right) & f_{1}\left(c\left(t_{2}\right)\right) & f_{0}\left(c\left(t_{2}\right)\right) \\
f_{2}\left(c\left(t_{i}\right)\right) & f_{1}\left(c\left(t_{i}\right)\right) & f_{0}\left(c\left(t_{i}\right)\right)
\end{array}\right|=0,
$$

for $i=3, \cdots, h d+1$ define the scheme $P\left(\Gamma_{\mathbb{L}}\right)$ for a small $\mathbb{L}$. Then

$$
\left(\frac{\widetilde{H^{0}\left(\Omega_{M}\right)}}{\mathcal{N}}\right) \otimes \mathcal{O}_{P\left(\Gamma_{\mathbb{L}}\right)} \simeq \Omega_{P\left(\Gamma_{\mathbb{L}}\right)}
$$

where $\widetilde{(\cdot)}$ denotes the sheaf associated to the module $(\cdot)$.

Therefore

$$
\begin{gathered}
\left(\frac{H^{0}\left(\Omega_{M}\right) \otimes k\left(c_{g}\right)}{\mathcal{N} \otimes k\left(c_{g}\right)}\right) \simeq \Omega_{P\left(\Gamma_{\mathbb{L}}\right)} \otimes k\left(c_{g}\right) \\
=\left.\left(\Omega_{P\left(\Gamma_{\mathbb{L}}\right)}\right)\right|_{\left(\left\{c_{g}\right\}\right)},
\end{gathered}
$$

where $k\left(c_{g}\right)=\mathbb{C}$ is the residue field at generic

$$
c_{g} \in P\left(\Gamma_{\mathbb{L}}\right) .
$$


Notice two sides of (2.119) are finitely dimensional linear spaces over $\mathbb{C}$.

$$
\begin{gathered}
\operatorname{dim}_{\mathbb{C}}\left(\left.\left(\Omega_{P\left(\Gamma_{\mathbb{L}}\right)}\right)\right|_{\left(\left\{c_{g}\right\}\right)}\right) \\
=\operatorname{dim}_{\mathbb{C}}\left(H^{0}\left(\Omega_{M}\right) \otimes k\left(c_{g}\right)\right)-\operatorname{dim}\left(\mathcal{N} \otimes k\left(c_{g}\right)\right)
\end{gathered}
$$

Since

$$
\begin{gathered}
\left.\operatorname{dim}_{\mathbb{C}}\left(\left.\left(\Omega_{P\left(\Gamma_{\mathbb{L}}\right)}\right)\right|_{\left(\left\{c_{g}\right\}\right)}\right)\right)=\operatorname{dim}\left(T_{c_{g}} P\left(\Gamma_{\mathbb{L}}\right)\right) \\
\operatorname{dim}\left(T_{c_{g}} P\left(\Gamma_{\mathbb{L}}\right)\right)=\operatorname{dim}(M)-\operatorname{dim}\left(\mathcal{N} \otimes k\left(c_{g}\right)\right) .
\end{gathered}
$$

By lemma 2.13,

$$
\operatorname{dim}\left(\mathcal{N} \otimes k\left(c_{g}\right)\right)=\operatorname{deg}(\omega)=h d-1 .
$$

The proposition 1.3 is proved.

Proof. of theorem 1.1: So by proposition 1.3,

$$
(n+1)(d+1)-(h d-1)
$$

is the dimension of the Zariski tangent space $T_{c_{0}} P\left(\Gamma_{\mathbb{L}}\right)$. Furthermore using lemma 2.8 par (b) and lemma 2.9 , we obtain that

$$
T_{c_{0}} \Gamma_{f_{0}}=(n+1)(d+1)-(h d-1)-2 .
$$

Again using lemma 2.8 part (a), we obtain that

$$
\operatorname{dim}\left(H^{0}\left(N_{c_{0} / X_{0}}\right)\right)=(n+1)(d+1)-h d-5 .
$$

Now we consider the exact sequence of sheaf modules on $\mathbf{P}^{1}$,

$$
0 \rightarrow N_{c_{0} / X_{0}} \rightarrow N_{c_{0} / \mathbf{P}^{n}} \rightarrow c_{0}^{*}\left(N_{X_{0} / \mathbf{P}^{n}}\right) \rightarrow 0 .
$$

This induces the exact sequence of finite dimensional linear spaces

$0 \rightarrow H^{0}\left(N_{c_{0} / X_{0}}\right) \rightarrow H^{0}\left(N_{c_{0} / \mathbf{P}^{n}}\right) \rightarrow H^{0}\left(c_{0}^{*}\left(N_{X_{0} / \mathbf{P}^{n}}\right)\right) \rightarrow H^{1}\left(N_{c_{0} / X_{0}}\right) \rightarrow 0$.

Using Eurler sequence for $\mathbf{P}^{n}$, we obtain

$$
\operatorname{dim}\left(H^{0}\left(N_{c_{0} / \mathbf{P}^{n}}\right)\right)=(n+1)(d+1)-4 .
$$

Using adjunction formula, we obtain

$$
\operatorname{dim}\left(H^{0}\left(c_{0}^{*}\left(N_{X_{0} / \mathbf{P}^{n}}\right)\right)=h d+1 .\right.
$$

Then by the calculations in (2.125), (2.128) and (2.129), we obtain

$$
\operatorname{dim}\left(H^{0}\left(c_{0}^{*}\left(N_{X_{0} / \mathbf{P}^{n}}\right)\right)=\operatorname{dim}\left(H^{0}\left(N_{c_{0} / \mathbf{P}^{n}}\right)\right)-\operatorname{dim}\left(H^{0}\left(N_{c_{0} / X_{0}}\right)\right)\right) .
$$

Hence by (2.127)

$$
\operatorname{dim}\left(H^{1}\left(N_{c_{0} / X_{0}}\right)\right)=0 .
$$

Theorem 1.1 in the Calabi-Yau case is proved. 


\subsection{Hypersurface of general type}

In this subsection, we prove theorem 1.1 for hypersurfaces of general type, i.e. the case $n+1-h<0$. So we let

$$
n+1+\delta=h
$$

where integer $\delta \geq 0$.

Let

$$
\operatorname{Pr}: \mathbf{P}^{n+\delta} \quad \rightarrow \quad \mathbf{P}^{n}
$$

be the projection. Then $\operatorname{Pr}$ induces a rational map

$$
\begin{array}{ccc}
\eta: \Gamma_{F_{0}} & -\rightarrow & \Gamma_{f_{0}} \\
c_{0}^{\delta} & \rightarrow & \operatorname{Pr} \circ c_{0} .
\end{array}
$$

Let $f_{0} \in H^{0}\left(\mathcal{O}_{\mathbf{P}^{n}}(h)\right)$ be generic for the $\mathbf{P}^{n}$ in (2.133). Let

$$
F_{0}=f_{0}+g_{0} \in H^{0}\left(\mathcal{O}_{\mathbf{P}^{n+\sigma}}(h)\right)
$$

be generic also. Let

$$
X_{0}=\operatorname{div}\left(f_{0}\right), Y_{0}=\operatorname{div}\left(F_{0}\right) .
$$

We may assume that $c_{0}^{\delta}$ lies in the regular locus of $\eta$, and $\eta\left(c_{0}^{\delta}\right)$ is not a constant map for generic $c_{0}^{\delta} \in \Gamma_{F_{0}}$. The map is clearly surjective. Then if $c_{0} \in \Gamma_{f_{0}}$ is generic, there is an inverse $c_{0}^{\delta}=\eta^{-1}\left(c_{0}\right)$ in $\Gamma_{F_{0}}$ which is also generic in $\operatorname{Hom}\left(\mathbf{P}^{1}, Y_{0}\right)$. By the result of section 2.1 ,

$$
H^{1}\left(N_{c_{0}^{\delta} / Y_{0}}\right)=0
$$

Let $K$ be the kernel bundle of the differential

$$
P r_{*}:\left.T_{\mathbf{P}^{n+\delta}}\right|_{R} \rightarrow T_{\mathbf{P}^{n}},
$$

where $R$ is the open set of $\mathbf{P}^{n+\delta}$ such that $\operatorname{Pr}$ restricted to $R$ is regular. So $K$ is a subbundle of $T_{R}$.

Now we have an exact sequence of bundles

$$
0 \rightarrow\left(c_{0}^{\delta}\right)^{*}(K) \rightarrow N_{c_{0}^{\delta} / Y_{0}} \rightarrow N_{c_{0} / X_{0}} \rightarrow 0
$$

Notice all bundles are over $\mathbf{P}^{1}$. Therefore we have the exact sequence of vector spaces

$$
H^{1}\left(N_{c_{0}^{\delta} / Y_{0}}\right) \rightarrow H^{1}\left(N_{c_{0} / X_{0}}\right) \rightarrow H^{2}\left(\left(c_{0}^{\delta}\right)^{*}(K)\right)=0 .
$$

By (2.137),

$$
H^{1}\left(N_{c_{0}^{\delta} / Y_{0}}\right)=0
$$

Hence

$$
H^{1}\left(N_{c_{0} / X_{0}}\right)=0 .
$$

This completes the proof of theorem 1.1. 


\section{Application 1: First encounter of Voisin's -Calabi-Yau hypersurfaces}

\subsection{Intuitive dimension count}

Our theorem rigorously proves that the following well-known dimension count is valid except for lower dimensions including K-3 surfaces. Consider all rational curves $C$ in a projective space of dimension $n$. There are $(n+1)(d+1)-1$ parameters in parameterizing rational curves of degree $d$ in $\mathbf{P}^{n}$. For a curve to be on a hypersurface of degree $h$, there will be $h d+1$ polynomial equation constraints on these parameters. Considering the 3 -dimensional automorphisms group of $\mathbf{P}^{1}$, to obtain a rational curve of degree $d$ on the hypersurface, naively we need to have

$$
(n+1)(d+1)-1-3 \geq h d+1 .
$$

For this inequality (3.1), we expect the strongest on generic hypersurfaces: all the mentioned $h d+1$ equations on the space of rational maps of dimension $(n+1)(d+1)-1$ are algebraically independent, i.e. they define a reduced variety of codimension $h d+1$. Our theorem 1.1 is just a rigorous proof of this expectation for $n \geq 4$. However our proof failed in the case $n \leq 3$ because of the vanishing differential form $\omega$. This leads to a fact that the intuitive inequality (3.1) does not hold for $n=3$ (the case of K-3 surfaces). The failure has no hint in the intuitive observation, but it is confirmed by Chen's result on rational curves on K-3 surfaces [1]. This exceptional case for $n=3$ distinguishes itself from other hypersurfaces because of their complex structures, and therefore it is in its own category.

\subsection{Voisin's conjecture on a Lang's conjecture}

In this section, we prove a Vosin's conjecture ([9], p. 114, after conjecture 3.22) which directly disproves a Lang's conjecture on the Abelian covering of a generic hypersurface. Voisin did not formally list it as a conjecture because this is a generalization of Clemens' conjecture.

(a) Vosin's conjecture (generalization of Clemens' conjecture): in a general CalabiYau hypersurface of dimension $\geq 3$, rational curves cover a countable union of Zariski closed proper algebraic subsets of codimension $\geq 2$.

(b) Lang's conjecture: if the general hypersurface $X_{0}$ is not of general type, the union of the images of non-constant rational maps: $A \rightarrow X$ from an Abelian variety $A$ to $X_{0}$ cover $X_{0}$.

In corollary 3.33, [9], Voisin proves that if (a) is correct, (b) is incorrect. In the following we prove (a) is correct. Therefore Lang's conjecture (b) is incorrect.

Corollary 3.1. Let $X_{0}$ be a Calabi-Yau general hypersurface of $\mathbf{P}^{n}, n \geq 4$. Let $m=\operatorname{dim}\left(X_{0}\right)-3$. Then the dimension of a parameter space of a family of rational curves on $X_{0}$ can only be $m$ at most.

Proof. Let $C_{0}$ be a rational curve and $c_{0}$ be its normalization. We may assume that $c_{0} \in \operatorname{Hom}\left(\mathbf{P}^{1}, X_{0}\right)$ is generic. By theorem 1.1, $H^{1}\left(N_{c_{0} / X_{0}}\right)=0$ for generic $c_{0} \in \operatorname{Hom}\left(\mathbf{P}^{1}, X_{0}\right)$. Since

$$
H^{1}\left(c_{0}^{*}\left(T_{\mathbf{P}^{1}}\right)\right)=0,
$$




$$
H^{1}\left(c_{0}^{*}\left(T_{X_{0}}\right)\right)=0
$$

By theorem 1.2, II.1 in [5],

$$
\operatorname{dim}_{\left[c_{0}\right]} \operatorname{Hom}\left(\mathbf{P}^{1}, X_{0}\right)=-K_{X_{0}} \cdot C_{0}+\operatorname{dim}\left(X_{0}\right) .
$$

Since $X_{0}$ is a Calabi-Yau, $c_{1}\left(K_{X_{0}}\right)=0$. Hence $K_{X_{0}} \cdot C_{0}=0$. We obtain that

$$
\operatorname{dim}_{\left[c_{0}\right]} \operatorname{Hom}\left(\mathbf{P}^{1}, X_{0}\right)=\operatorname{dim}\left(X_{0}\right) .
$$

Since there is a 3-dimensional group of automorphisms on $\mathbf{P}^{1}$, our corollary is proved.

This immediately implies the conjecture (a), i.e.

COROLlary 3.2. Rational rational curves on a general Calabi-Yau hypersurface of dimension $\geq 3$ cover a countable union of Zariski closed proper algebraic subsets of codimension $\geq 2$.

Proof. Because

$$
\operatorname{dim}_{\left[c_{0}\right]} \operatorname{Hom}\left(\mathbf{P}^{1}, X_{0}\right)=\operatorname{dim}\left(X_{0}\right),
$$

the dimension of the parameter space of a family of rational curves has to be

$$
\operatorname{dim}\left(X_{0}\right)-3 .
$$

Then each irreducible uniruled variety must have dimension at most

$$
\operatorname{dim}\left(X_{0}\right)-2 .
$$

The corollary is proved.

Remark. In the case of $\operatorname{dim}\left(X_{0}\right)=3$, corollary 3.2 is the Clemens' conjecture which is proved in [10].

Corollary 3.3. For each $d \geq 1$, the generic hypersurface $X_{0}$ of degree $2 n-3$ in $\mathbf{P}^{n}, n \geq 4$, contains at most a finitely number of rational curves of degree $d$.

Proof. Using the formula (3.2), we obtain that

$$
\begin{gathered}
\operatorname{dim}_{\left[c_{0}\right]} \operatorname{Hom}\left(\mathbf{P}^{1}, X_{0}\right)-3=-K_{X_{0}} \cdot C_{0}+\operatorname{dim}\left(X_{0}\right)-3 . \\
=(n-4)(1-d) \leq 0 .
\end{gathered}
$$

Considering the 3-dimensional group of automorphisms on $\mathbf{P}^{1}$, our corollary is proved.

Remark The corollary for the case $n \geq 5$ is also a consequence of Voisin's theorem in [7] (see [6]), even though her approach is quite different from ours. The corollary for $n=4$ is one of Calabi-Yau cases above, which also is the Clemens' conjecture. 


\section{Application 2: Second encounter of Vosin's -hypersurfaces of general type}

\subsection{Work by Clemens, Voisin and Pacienza}

Clemens, Voisin, Pacienza and others studied questions about genus of subvarieties of hypersurfaces. Consequently they obtain many results of rational curves on hypersurfaces. Theorem 1.1 clearly is different from their general questions on genus of subvarieties, but it has many corollaries that not only coincide with their inequalities but also go beyond them.

We first give a bound of degree of hypersurfaces.

Corollary 4.1. Let $X_{0}$ be a general hypersurface of $\mathbf{P}^{n}, n \geq 4$ that contains an irreducible rational curve $C_{0}$ of degree $d$, then

$$
\operatorname{deg}\left(X_{0}\right) \leq n+1+\frac{n-4}{d} .
$$

Proof. Let $h=\operatorname{deg}\left(X_{0}\right)$. Let $c_{0}: \mathbf{P}^{1} \rightarrow C_{0}$ be a birational map (normalization of $\left.C_{0}\right)$. We may assume that $c_{0} \in \operatorname{Hom}\left(\mathbf{P}^{1}, X_{0}\right)$ is generic. Because $n \geq 4$,

$$
H^{1}\left(N_{c_{0} / X_{0}}\right)=0 .
$$

Next we see that a numerical meaning of $H^{1}\left(N_{c_{0} / X_{0}}\right)=0$ is the inequality (4.1).

This follows from the observation:

$c_{0}^{*}\left(T_{X_{0}}\right)$ is a locally free sheaf over $\mathbf{P}^{1}$. Thus it has splitting

$$
c_{0}^{*}\left(T_{X_{0}}\right) \simeq \mathcal{O}_{\mathbf{P}^{1}}\left(a_{1}\right) \oplus \cdots \oplus O_{\mathbf{P}^{1}}\left(a_{n-1}\right) .
$$

Consider the exact sequence

$$
0 \rightarrow T_{\mathbf{P}^{1}} \rightarrow c_{0}^{*}\left(T_{X_{0}}\right) \rightarrow N_{c_{0} / X_{0}} \rightarrow 0 .
$$

We then have

$$
0=H^{1}\left(T_{\mathbf{P}^{1}}\right) \rightarrow H^{1}\left(c_{0}^{*}\left(T_{X_{0}}\right)\right) \rightarrow H^{1}\left(N_{c_{0} / X_{0}}\right) .
$$

Because $H^{1}\left(N_{c_{0} / X_{0}}\right)=0, H^{1}\left(c_{0}^{*}\left(T_{X_{0}}\right)\right)=0$. Hence

$$
a_{i} \geq-1, \text { for all } i=1, \cdots, n-1 \text {. }
$$

Notice the tangential automorphisms which lie in $H^{0}\left(c_{0}^{*}\left(T_{X_{0}}\right)\right)$ have two zeros. This means that at least one of $a_{i}, i=1, \cdots, n-1$ is larger than or equal to 2 . This shows

$$
c_{1}\left(c_{0}^{*}\left(T_{X_{0}}\right)\right) \geq-n+4 .
$$

By the adjunction formula

$$
(n+1-h) d=c_{1}\left(c_{0}^{*}\left(T_{X_{0}}\right)\right) .
$$


Hence

$$
(n+1-h) d \geq-n+4 .
$$

This is the inequality (4.1).

Clemens started the early study of rational curves on hypersurfaces in [2]. Later Voisin and Pancienza borrowed Ein's original idea of using adjunction formula and pushed it further to obtain the minimum genus of subvaries in hypersurfaces [6], [7]. Their immediate application of it is for rational curves. They obtained the following results

(1) (Clemens and Voisin) The maximum degree of a generic hypersurface of $\mathbf{P}^{n}$ for $n \geq 4$ containing a rational curve is

$$
2 n-3
$$

and the maximum degree can be achieved ( see [2], [7]).

(2) (Pancienza) A generic hypersurface of $\mathbf{P}^{n}$ of degree $2 n-3$ for $n \geq 6$ does not contain rational curves of degree larger than 1 (see [6]).

Our inequality confirms their results and indicates that their numerical results are originated from

$$
H^{1}\left(N_{c_{0} / X_{0}}\right)=0 \text {. }
$$

This is because if $d \geq 1$, then for all $n \geq 4$ the inequality (4.1) reduces to

$$
\operatorname{deg}\left(X_{0}\right) \leq n+1+n-4=2 n-3 .
$$

This is the maximum degree given by Voisin. In case $h=2 n-3$ and if $n$ is strictly larger than 3 , the inequality (4.1) becomes $d \leq 1$. This means that such a general hypersurface $(n \geq 5)$ does not contain rational curves other than lines. In the case $n \geq 6$, this is a result (2) by Pacienza. Our result shows that this is also true for $n=5$.

The inequality (4.1) is the intuitive inequality (3.1).

\subsection{Sharp bounds of degrees of rational curves on general hypersur- faces of general type}

It is clear that when $X_{0}$ is Fano or Calabi-Yau, i.e., $h \leq n+1$, the inequality (4.1) does not produce anything new. But the situation is well understood. If $X_{0}$ is Fano, it is rationally connected. If it is Calabi-Yau, the situation is discussed in section 3.2. The only remaining case is when $X_{0}$ is of general type. In this case, there is a conjecture by Voisin ( conjecture 3.9, [9]): if a generic hypersurface is of general type, the degrees of rational curves on it are bounded. Our inequality (4.1) shows that the conjecture is true, and furthermore we give the sharp bound:

COROLlary 4.2. If $X_{0}$ is a general hypersurface of $\mathbf{P}^{n}, n \geq 4$ of general type, i.e., $h>n+1$, then the degrees of rational curves $C_{0}$ have an upper bound

$$
\operatorname{deg}\left(C_{0}\right) \leq \frac{n-4}{h-n-1}
$$


Proof. If $h>n+1$ (i.e. it is of general type), the inequality (4.5) is exactly the inequality (4.1).

Above inequalities (4.1) and (4.5) describe a complete picture of rational curves on a general hypersurface $X_{0}$ of general type in $\mathbf{P}^{n}, n \geq 4$. We would like to describe them separately because historically some of them were not obtained as one unified result from $H^{1}\left(N_{c_{0} / X_{0}}\right)=0$, but rather as individual results with other methods.

(1) if $h$ is in the interval $(2 n-3,+\infty)$, the inequality (4.1) for $d \geq 1$ implies that there are no rational curves on $X_{0}$. This is the known result of Clemens ([2]) and Voisin ([7], [8]) ;

(2) if $h=2 n-3$, and $n \geq 5$ the inequality (4.1) implies that $X_{0}$ contains no rational curves other than lines. Pacienza in ([6]) obtained this result for $n \geq 6$;

(3) if $h$ is in $\left(\frac{3 n}{2}-1,2 n-3\right)$, the inequality (4.5) implies that $X_{0}$ does not contain rational curves other than lines. This is the known result of Clemens and Ran ([3]), which is implied by their bound of twisted genus;

(4) if $h=\frac{3 n}{2}-1$, the inequality (4.5) implies that $X_{0}$ contains no rational curves other than lines and quadratic curves. This is our new result.

(5) if $h$ is in $\left(n+1, \frac{3 n}{2}-1\right)$, the degrees of the rational curves $C_{0}$ are bounded above as in the formula (4.5). This is our new result.

\section{REFERENCES}

[1] X. Chen, Rational curves on K-3 surfaces, Arxiv math/9804075 1, April 15, 1998.

[2] H. Clemens, Curves in generic hypersurfaces, Ann. Sci. École Norm. Sup. 19(1986), pp. 629636

[3] H. Clemens And Z. RAn, Twisted genus bounds for subvarieties of generic hypersurfaces, American Journal of Mathematics 126(2004), pp. 89-120.

[4] J. Harris, M. Roth and J. Star, Rational curves on Hypersurfaces of low degree, J. Reine Angew. Math., 571 (2004), pp. 45-96.

[5] J. KolLÁr, Rational curves on algebraic varieties, Springer (1996)

[6] G. Pacienza, Rational curves on general projective hypersurfaces, J. Algebraic Geometry, 12 (2003), pp. 471-476.

[7] C. Voisin 1, On a conjecture of Clemens on rational curves on hypersurfaces, J. Dfferential Geometry, 44(1996), pp. 200-213.

[8] C. Voisin 2, A correction: "On a conjecture of Clemens on rational curves on hypersurfaces", J. Dfferential Geometry 49(1998), pp. 601-611.

[9] C. Voisin 3, On some problems of Kobayashi and Lang: Algebraic approaches, International Press, Current developments in mathematics (2003), pp. 53-125.

[10] B. WANG, Rational curves on generic quintic threefolds, arXiv:1202.2831 2014 